\title{
Thermodynamics of the Air-Water Interface of
} Mixtures of Surfactants with Polyelectrolytes, Oligoelectrolytes and Multivalent Metal

\section{Electrolytes}

\author{
Robert K.Thomas ${ }^{*, \dagger}$ and J.Penfold \\ †Physical and Theoretical Chemistry Laboratory, South Parks Road, Oxford, OX1 3QZ, \\ United Kingdom \\ $\ddagger S T F C$, Rutherford-Appleton Laboratory, Chilton, Didcot, Oxfordshire, OX11 ORA, United \\ Kingdom
}

E-mail: robert.thomas@chem.ox.ac.uk 


\begin{abstract}
A full comparison of results from binding isotherms and surface tension (ST) measurements on polyelectrolyte (PE)-surfactant (S) mixtures, especially the polymer dependence, shows up clear distinctions between the behaviour of two representative PES systems, poly(sodium styrenesulfonate) (NaPSS) with dodecyltrimethylammonium bromide $\left(\mathrm{C}_{12} \mathrm{TAB}\right)$ and poly(dimethyl-diallyl-ammonium chloride) (PDMDAAC) with sodium dodecylsulfate (SDS) in $100 \mathrm{mM} \mathrm{NaCl}$. The surfactant-monomer binding constant in NaPSS- $\mathrm{C}_{12}$ TAB is an order of magnitude greater than that in the PDMDAACSDS-NaCl system. This results in the ST behaviour being dominated largely by noncooperativity in the former and by cooperativity in the latter. This leads to the ST in PDMDAAC-SDS-NaCl being at its lowest when the average bound fraction is low, but increasing rapidly as saturation approaches. A full analysis is also given of how this is altered in the mixture of PDMDAAC-SDS-NaCl with the nonionic surfactant hexethylene glycol monododecyl ether. In contrast, the much stronger interaction in NaPSS $-\mathrm{C}_{12}$ TAB leads to high ST complexes at low bound fractions while the lowest ST occurs near the maximum bound fraction, close to or at precipitation. In the PDMDAAC-SDS-NaCl system the ST drops to a low value at low surfactant concentrations but then increases to a high value just before precipitation occurs, which, combined with increasing surface adsorption of free surfactant, results in a sharp ST peak. In the NaPSS- $\mathrm{C}_{12} \mathrm{TAB}$ system the $\mathrm{ST}$ does not drop until the system is at or close to precipitation and it then stays on a plateau until the point at which free surfactant takes over. Thermodynamics does not allow large step changes in the ST and it is suggested that where these have been observed they are the result of self-depletion of $\mathrm{PE}$ or $\mathrm{PE}-\mathrm{S}$ complexes on the finely divided precipitate of the complex and are therefore not representative of full equilibrium.
\end{abstract}




\section{Introduction}

When an ionic surfactant is added to a solution containing a multicharged electrolyte surface active complexes tend to form at low concentrations by interaction of the surfactant ion with the oppositely multicharged ion, and this results in a low surface tension (ST) of the air-water interface. The reaction is driven by electrostatic interactions between the charged groups, hydrophobic interactions between the surfactant chains, entropic contributions from the ions and, in the case of a polyelectrolyte, hydrophobic contributions from groups on the multicharged ion. Examples vary from the interaction of sodium alkylethoxysulfates (SAES) with $\mathrm{AlCl}_{3},{ }^{1}$ to the interaction of sodium dodecyl sulfate (SDS) with either small amines ${ }^{2}$ or with cationic polymers such as poly(dimethyldiallyammonium chloride) (PDMDAAC). ${ }^{3}$ The strong depression of the ST caused by these complexes at low surfactant concentrations makes them valuable in many surface applications. At higher concentrations of surfactant the ST behaviour is often complicated by precipitation and further complexation before excess surfactant causes redissolution back to a single solution whose ST will then be dominated by the high concentration of surfactant. Precipitation, especially where it involves polyelectrolytes, may adversely affect equilibration in these systems. Of particular concern has been the effect of colloid formation in the precipitation step, which can lead to a metastable suspension instead of redissolution. ${ }^{4,5}$

A quantitative account of the surface behaviour at the air-water interface requires an understanding of the combined effects of a reacting mixture and phase separation on the ST. There has been much discussion of the complicated ST behaviour relative to that of simple surfactant solutions. Buckingham showed that the initial drop in the ST at low concentrations can often be accounted for by assuming that the Gibbs equation for the simple surfactant applies. ${ }^{6,7}$ This is result of cancellation of terms involving the stoichiometry of the polyelectrolyte-surfactant complex $(\mathrm{PE}-\mathrm{S})$ in the total coverage and the Gibbs prefactor. Two models for the wider range of ST behaviour have been proposed. The first, by Bell et al. ${ }^{8,9}$ is a simple mass action model that shows how the ST can increase with surfac- 
tant concentration over a certain range by preferential solubilization of polymer into micelles. The second, by Bahramian et al., ${ }^{10}$ is a thermodynamic model based on the Butler equation, which rationalizes the full range of the observed behaviour in terms of a series of fixed points, whose characteristics are fitted to the data. Both these models assume thermodynamic equilibration of the system, the former with only pseudophase (micellar) separation rather than full phase separation, but the latter includes precipitation. Campbell et al. have focussed on non-equilibrium aspects of the problem but have recently proposed an equilibrium model for the peak in the ST for two different types of PE-S mixture, in which the peak is attributed to the complete precipitation of the polyelectrolyte with the ST being solely determined by the residual surfactant. ${ }^{11}$

Neutron reflection (NR) has separately identified a unexpected range of multilayer structures adsorbed at the air-water interface, the first of which was for a mixture of polystyrene sodium sulfonate with dodecyltrimethylammonium bromide (NaPSS- $\mathrm{C}_{12} \mathrm{TAB}$ ), for which Taylor et al. discussed at length the question of whether the multilayer (monolayer + bilayer) was at equilibrium, but without reaching a definite conclusion other than it was at least an extremely stable steady state. ${ }^{12}$ Multilayer structures have since been observed in a wider range of systems ${ }^{13}$ including ones not involving polymers or phase transitions, most notably in multivalent ion-surfactant systems, $\mathrm{M}^{n}-\mathrm{S}$, where $n$ denotes the charge. ${ }^{1,14-16}$ These are simpler systems, often showing a well defined evolution of structure with composition, and almost certainly at equilibrium. They therefore offer new opportunities for understanding the more complex PE-S systems. Given the technological value of low ST systems, it is important to understand both equilibrium and kinetic properties of $\mathrm{PE}-\mathrm{S}$ systems. However, since kinetic behaviour can usually only be explained within an underlying thermodynamic framework, characterization of the equilibrium behaviour is ultimately more important. The aim of this paper is therefore to establish some rules for assessing equilibration of the airwater interface in these complex systems, and to use new insight from the simpler $\mathrm{M}^{n}-\mathrm{S}$ systems to extend the earlier thermodynamic models of Bahramian et al. and Bell et al. 


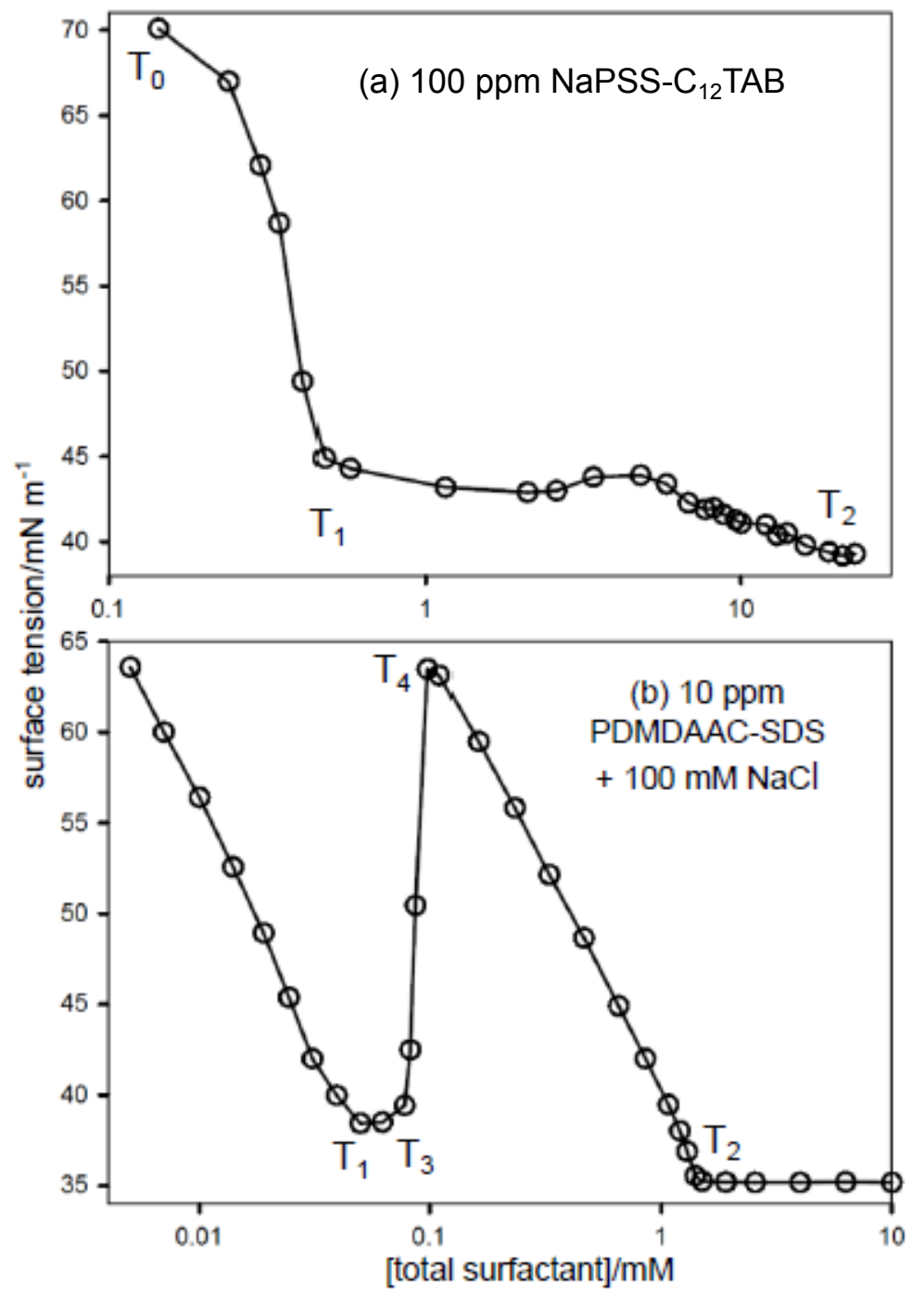

Figure 1: Examples of measured surface tension curves of (a) NaPSS- $\mathrm{C}_{12} \mathrm{TAB}^{12}$ and (b) PDMDAAC-SDS $(+100 \mathrm{mM} \mathrm{NaCl}) .{ }^{3}$ Key features are labelled $\mathrm{T}_{0}$ (onset of the initial fall in ST), $\mathrm{T}_{1}$ (onset of the initial plateau in the ST), $\mathrm{T}_{2}$ (onset of the final ST plateau), $\mathrm{T}_{3}$ (onset of a peak in the $\mathrm{ST}$ ), and $\mathrm{T}_{4}$ (top of the peak in the $\mathrm{ST}$ ). $\mathrm{T}_{0}$ is off scale for PDMDAAC-SDS and is not shown. Not all PE-S curves are as clearly defined as the two shown here. 
In principle, the ultimate test of equilibration at the air-water interface is to demonstrate that the Gibbs equation can be quantitatively fitted to the data. However, this requires more detail about the surface composition than is normally available. Although NR does offer some solutions to this problem, it has only so far been applied in relatively simple cases. ${ }^{17-19}$ The full application of the Gibbs equation to $\mathrm{PE}-\mathrm{S}$ systems is therefore not a practicable test, and we have to look for alternatives. We use the $\mathrm{M}^{n}-\mathrm{S}$ systems to explore the simpler issues. We then focus particularly on the PDMDAAC-SDS and NaPSS- $\mathrm{C}_{12}$ TAB systems mentioned above, which represent two extremes of behaviour and for which there are sets of data from a range of different experiments. It is useful to have easy identification of key parts of the ST curves of these two systems and for this purpose two original examples of their ST behaviour are shown with points marked $\mathrm{T}_{0}, \mathrm{~T}_{1}$, etc. in Figure 1 . We discuss other sets of measurements on the same systems, which differ in greater or lesser respects, in the body of the paper.

\section{Surface Tension and Complexation}

In a simple mixture of two surfactants the surface is often different from the overall bulk composition and Bahramian et al. have drawn attention to the complications in the surface behaviour of PE-S systems that may result from this difference. ${ }^{10}$ To discuss the complexes in $\mathrm{PE}-\mathrm{S}$ and $\mathrm{M}^{n}-\mathrm{S}$ systems we use the nomenclature $\mathrm{P}^{n}$ and $\mathrm{M}^{n}$ for the $n$-valent polyion and metal ion, and R, S, M and X for polyion monomer, surfactant ion and metal and other counter ion respectively, when it is necessary to distinguish these terms, but we retain PES for the less well defined polyelectrolyte surfactant complex. Surfactant ions bind to the polyion individually through electrostatic or entropic interactions. Non-cooperative binding (N-binding) can be approximately described by a Langmuir isotherm, for which the binding energy is the same for all sites, whether on the same or different PE molecules. Cooperative binding (C-binding) is driven mainly by hydrophobic interactions between the surfactant chains in concert with the electrostatic interactions, and may be assisted or opposed by the 
conformational energy of the PE chain and the hydrophobicity of the monomer fragments. $\mathrm{N}-$ and $\mathrm{C}$-binding are combined in an isotherm proposed by Satake and Yang, ${ }^{20}$ which is based on the Zimm-Bragg model of the helix-coil transition in $\mathrm{DNA}^{21}$ and is

$$
\phi=\frac{1}{2}\left[1+\frac{K c_{f}-1}{\sqrt{\left(1-K c_{f}\right)^{2}+\frac{4 K c_{f}}{\alpha}}}\right]
$$

where $\phi$ is the fractional binding, $K$ is the binding constant, $c_{f}$ is the concentration of free surfactant and $\alpha$ is a cooperativity factor. At the limit of no cooperativity $\alpha=1$ and the equation reduces to the Langmuir isotherm. The single cooperative term is not always adequate to describe the range of cooperativity exhibited by PE-S systems, but here we use it to make more qualitative distinctions between the two systems, where this inadequacy is not important.

Several binding curves, with Satake-Yang fits, plotted against free surfactant concentration are shown for NaPSS- $\mathrm{C}_{12}$ TAB in Figure 2. The data are for $100 \mathrm{ppm}$ NaPSS- $\mathrm{C}_{12}$ TAB in $21 \mathrm{mM} \mathrm{NaCl}^{22}$ and 1000 ppm PSS- $\mathrm{C}_{12}$ TAB in $10 \mathrm{mM} \mathrm{NaCl}$ plotted against $[\mathrm{S}] / 10 .{ }^{23,24} \mathrm{~A}$ third calculated curve for 100 ppm NaPSS- $\mathrm{C}_{12}$ TAB in $10 \mathrm{mM} \mathrm{NaCl}$ (dashed line) is based on parameters of Hayakawa and Kwak (HK) which they used to fit data that was not shown in their paper. ${ }^{22}$ For the data in $21 \mathrm{mM} \mathrm{NaCl}$ an optimized fit to the cooperative region of binding $(\alpha=200)$ is shown as a continuous line and a dotted line shows a very approximate fit of the Langmuir isotherm $(\alpha=1)$ to the binding at higher concentrations. HK showed that the curves change little in shape as the concentration of $\mathrm{NaCl}$ increases but the binding constant $\mathrm{K}$ decreases strongly. Another important feature of the NaPSS- $\mathrm{C}_{12} \mathrm{TAB}$ system is that the binding curves shift in concentration by a factor proportional to the polymer concentration. ${ }^{24}$ This is demonstrated in Figure 2 by the 1000 ppm data of Almgren et al. which closely fits the 100 ppm curve of HK when the bound fraction is plotted against [S]/10 instead of [S]. Thus, in terms of Eqn (1) the parameter $K$ can be accurately represented by $K^{\prime} /[\mathrm{R}]$ where $[\mathrm{R}]$ is the molar segment concentration. Hansson and Almgren interpreted this 


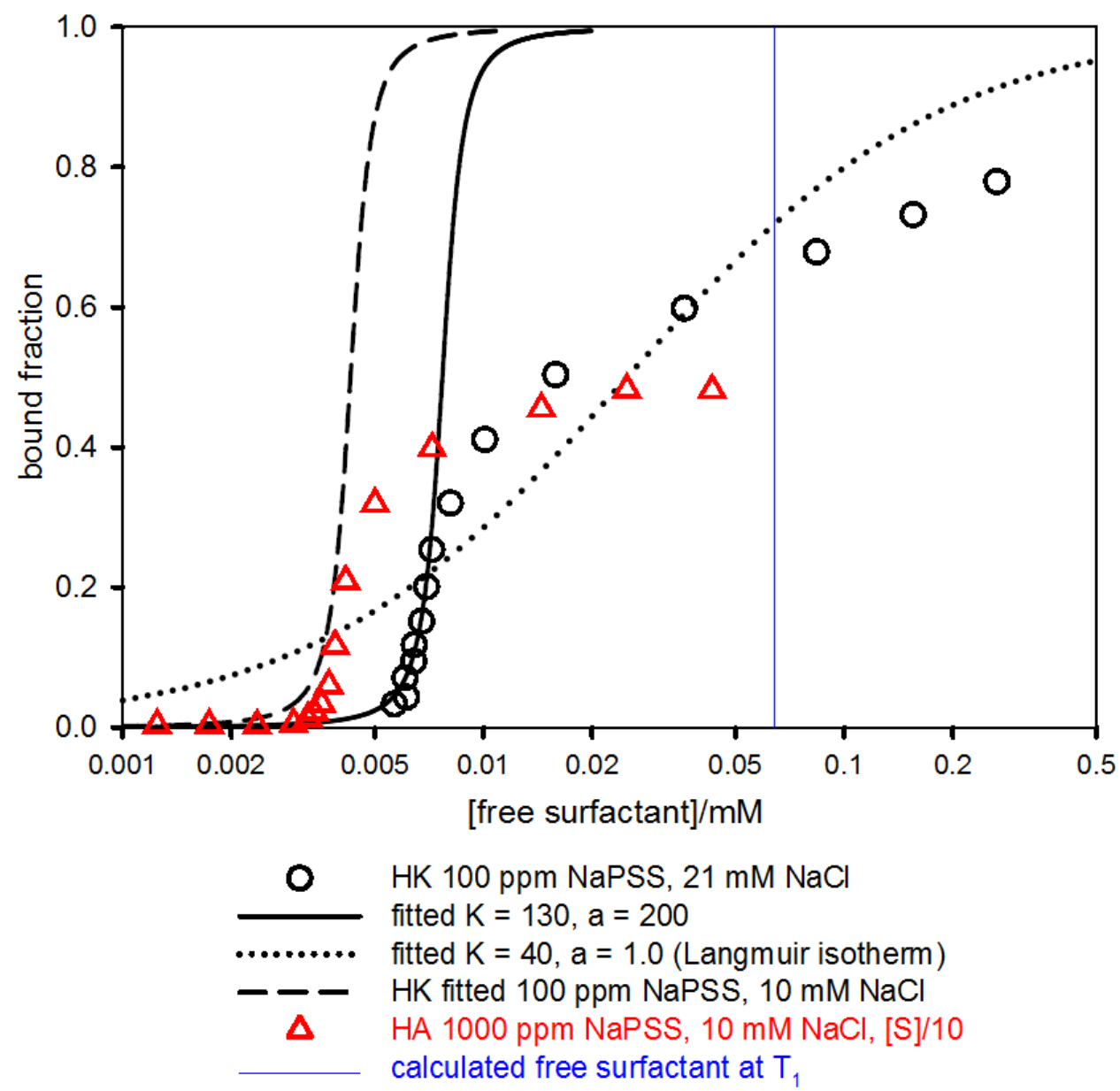

Figure 2: Experimental and fitted binding curves for NaPSS- $\mathrm{C}_{12} \mathrm{TAB}$ as a function of free surfactant concentration. Experimental data are shown for 100 ppm NaPSS in $20 \mathrm{mM} \mathrm{NaCl}$ (from Hayakawa and Kwak (HK) ${ }^{22}$ ) and $1000 \mathrm{NaPSS}$ in $10 \mathrm{mM} \mathrm{NaCl}$ plotted against $[\mathrm{S}] / 10$ (from Almgren et al. $(\mathrm{HA})^{23}$ ). Fits are shown for different parameters in the Satake-Yang equation. A calculated curve is shown for $100 \mathrm{ppm} \mathrm{NaPSS}$ in $10 \mathrm{mM} \mathrm{NaCl}$ using parameters from HK. The free surfactant concentration at $\mathrm{T}_{1}$ (see Figure 1) for $\mathrm{NaPSS}_{-} \mathrm{C}_{12} \mathrm{TAB}$ as determined from Figure 6 is shown as a vertical line with the shaded area indicating the error. 
as resulting from initial $\mathrm{N}$-binding of individual surfactant molecules, which has to reach a threshold bound fraction before C-binding to give attached micelles can begin. At 100 ppm NaPSS ([R] of $0.048 \mathrm{mM}$ ) the onset of cooperativity is at about $0.005 \mathrm{mM}$, i.e. about $10 \%$ of the NaPSS charged sites must be occupied before C-binding starts. In Figure 2 we also show the estimated free surfactant concentration at $T_{1}$, determined as described below, which marks the onset of the ST plateau. This shows that neither the initial N-binding of surfactant nor most of the $\mathrm{C}$-binding range leads to species that lower the ST in this system, a feature that had previously been clearly identified by Monteux et al. ${ }^{25}$

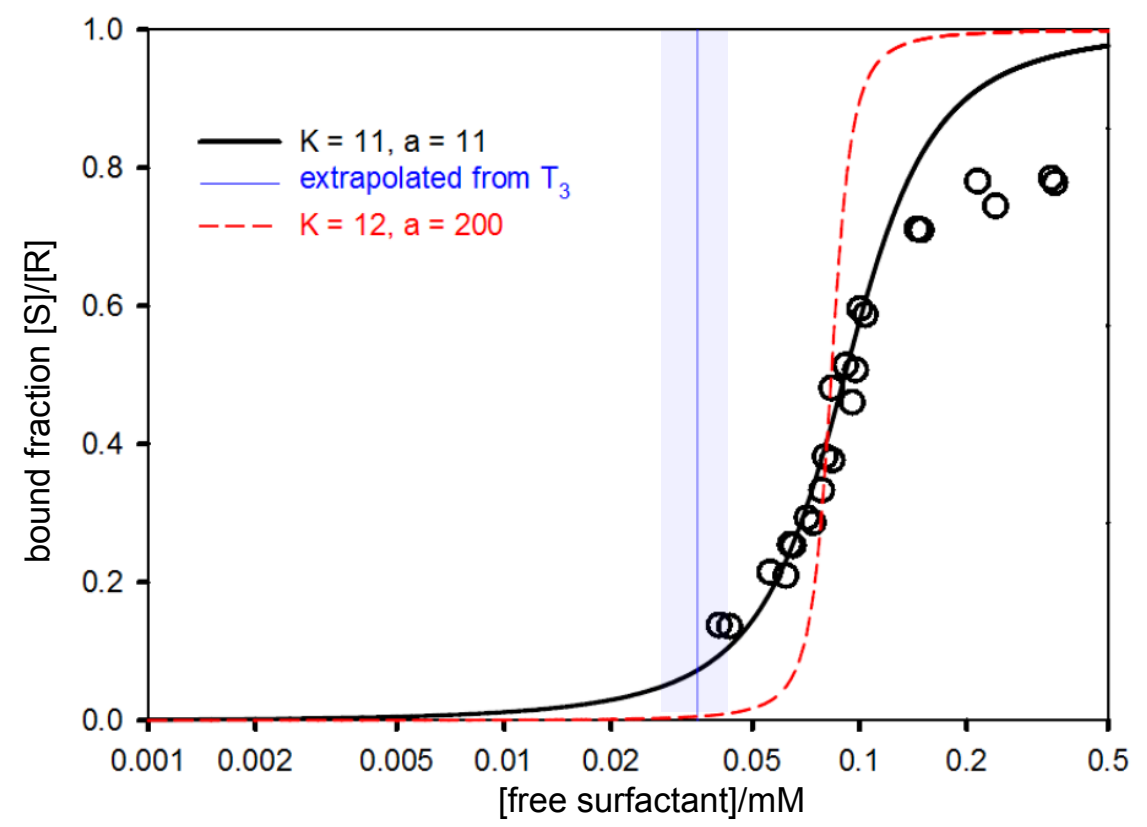

Figure 3: Experimental and fitted binding curves for $100 \mathrm{ppm}$ PMDAAC-SDS in $0.1 \mathrm{M}$ $\mathrm{NaCl}$ (data from Nizri et al. ${ }^{26}$ ) as a function of free surfactant concentration. The calculated binding curves use Eqn (1). The free surfactant concentrations at $\mathrm{T}_{3}$ for PDMDAAC-SDS$\mathrm{NaCl}(100 \mathrm{mM})$ as determined from Figure 6 are shown as vertical lines with the shaded area indicating the error.

Figure 3 shows the binding curve for our other main example, PDMDAAC-SDS in 100 $\mathrm{mM} \mathrm{NaCl} .{ }^{26}$ The main part of the curve shows moderate cooperativity. The striking feature is that it is now the initial binding region that leads to a low ST so that, although the binding constant is an order of magnitude weaker than for NaPSS- $\mathrm{C}_{12} \mathrm{TAB}$, the onset of the low ST 
plateau in the two systems is at about the same surfactant concentration. The variation of this isotherm with polymer concentration in $100 \mathrm{mM} \mathrm{NaCl}$ has not been studied but, in contrast to the binding in NaPSS- $\mathrm{C}_{12}$ TAB at low concentrations, the binding in PDMDAACSDS (in $1 \mathrm{mM} \mathrm{NaCl}$, i.e. very little electrolyte) is independent of polymer concentration over the range 100-400 ppm and is more cooperative than in $100 \mathrm{mM} \mathrm{NaCl} .{ }^{26-28}$ Indirect information on the effect of polymer concentration in $100 \mathrm{mM} \mathrm{NaCl}$ comes from the ST curves shown in Figure 5(b). Thus, at an ST of about $42 \mathrm{mN} \mathrm{m}^{-1}$ the curves at four different polymer concentrations pass through a point where they all have the same ST at the same overall surfactant concentration. This requires that the bound fraction at that concentration does not change with polymer concentration, which is again only compatible with C-binding. Furthermore, since the constant ST at this point is low these C-bound complexes are strongly surface active.

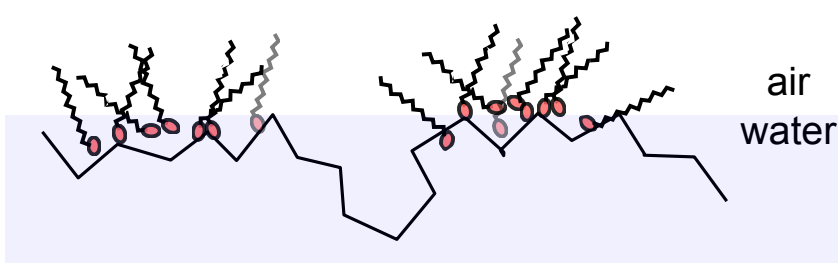

(a) C-binding

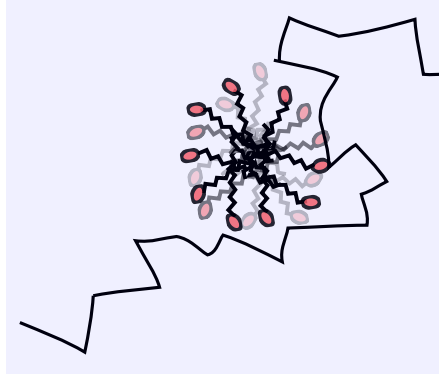

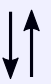

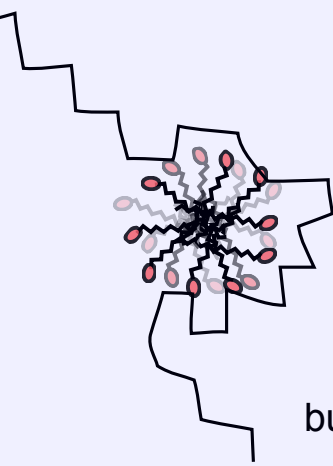

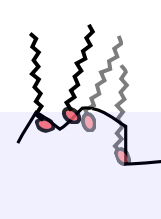

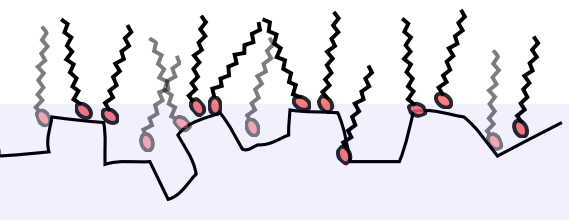

$\downarrow \uparrow$ (b) N-binding

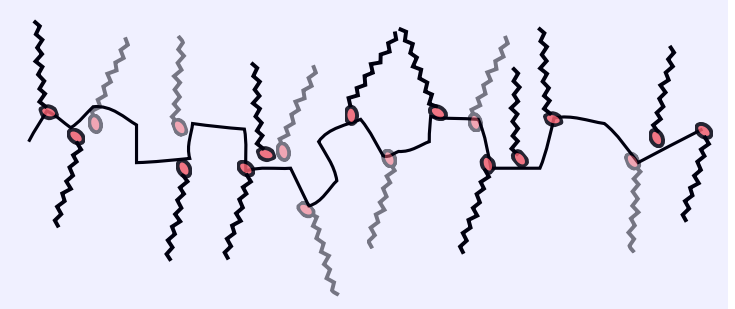

bulk solution

Figure 4: Schematic diagrams of different types of binding in PE-S complexes in the bulk and at surfaces. The terms $\mathrm{C}-$ and $\mathrm{N}$-binding are explained in the text.

It is helpful to assess how $\mathrm{N}$ - and $\mathrm{C}$-binding might influence the surfac tension. Figure 4 gives a schematic sketch of possible complexes in bulk and at the surface. The simple 
structures shown suggest that N-binding is generally more likely to favour lowr STs because the surfactant molecules $(\mathrm{S})$ can attach to the PE in a way that favours the hydrophobicity of the S chains while allowing the complex to extend at the surface. C-binding, on the other hand, favours bulk complexes because it shields the $\mathrm{S}$ chains from water by the formation of aggregates. However, the opposite seems to be occurring in our two main examples. At low $\mathrm{S} / \mathrm{PE}$ ratios a uniform distribution of $\mathrm{S}$ on the segments in $\mathrm{NaPSS}-\mathrm{C}_{12} \mathrm{TAB}$ is driven by a large binding constant, ${ }^{29}$ which controls the individual interaction of $\mathrm{S}$ with $\mathrm{PE}$ binding sites. However at low [S] the complexes contain too little S to lower the ST significantly, even though adsorption is favoured by the $\mathrm{N}$-binding. For PDMDAAC-SDS the $\mathrm{C}$-binding that causes high stoichiometry complexes to be formed in preference to a uniform distribution of $\mathrm{S}$ amongst the PE molecules is helped by the weaker individual interactions of $\mathrm{S}$ with PE binding sites, i.e. by the lower $K{ }^{29}$ The Gibbs equation, however, shows that the ST behaviour is driven not just by an intrinsically low ST of the complete layer but also by a high concentration of the surface active complex in the bulk solution. C-binding can result in a significant concentration of complexes with a high enough $\mathrm{S}-\mathrm{PE}$ ratio to be surface active. These then have the potential to lower the ST if they can rearrange themselves at the surface. A possible rearrangement is illustrated schematically in Figure 4(a). To show how it can occur we use a thermodynamic device of Thomson. ${ }^{30}$

The formation of a complex in the bulk solution can be written in terms of $\mathrm{R}$ and $\mathrm{S}$ by

$$
\mathrm{R}+n \mathrm{~S} \leftrightarrows \mathrm{RS}_{n}
$$

and it is governed by an equilibrium constant $K_{n}$ where $R T \ln K_{n}=-\Delta G_{0}$ where $\Delta G_{0}$ includes all the bulk free energy terms from the Satake-Yang equation, but no surface terms. We then define a different equilibrium constant $K_{n}^{\sigma}$ by

$$
R T \ln \frac{K_{n}^{\sigma}}{K_{n}}=-[\Delta(a \sigma)+\Delta \beta]
$$


where $a$ is the molecular area, $\sigma$ is the surface tension, $\beta$ is a measure of cooperative binding energy, and $\Delta$ represents changes between bulk solution and surface. Although this concept of the surface region has not been defined in the framework of the Gibbs formulation, it has sufficient validity for the semi-quantitative explanation of differences between surface and bulk compositions. Thus, a negative value of $\Delta(a \sigma)$ for a complex of given stoichiometry will shift $K_{n}^{\sigma}$ in favour of that complex at the surface. Per surfactant the maximum estimated effect on the surface energy is an increase of an order of magnitude in $K_{n}^{\sigma} / K_{n}$ (based on $a=40 \AA^{2}$ and $\sigma=45 \mathrm{mN} \mathrm{m}^{-1}$ ). This is large enough to cause a low ST even when the adsorbed complex is only present at low concentration in the bulk solution. If C-binding is strong enough for a complex with high surfactant stoichiometry to be present at significant concentration in the bulk solution, adsorption then only requires a small gain in surface energy. Thus, in Figure 4(a), although C-binding energy is lost (positive $\Delta \beta$ ) on moving from bulk to surface a relatively small gain in surface energy is sufficient to offset this loss and ensure a low ST. This is evidently the situation for PDMDAAC-SDS at a low average bound fraction.

The ST behaviour of PE-S systems is measured as a function of total surfactant, whereas the binding curves are measured in terms of the free surfactant in the system. The free surfactant concentration for the approximate onset of the low ST plateau in Figures 2 and 3 was extracted from the variation of ST with polymer concentration by using the ST plots at different polymer concentrations shown in Figure 5. Certain features are well defined and vary systematically with polymer concentration, e.g. $\mathrm{T}_{1}$ for NaPSS- $\mathrm{C}_{12} \mathrm{TAB}$, and $\mathrm{T}_{3}$ for PDMDAAC-SDS, for the reasons given above. Since the ST depends on the monomer activity (approximately monomer concentration) it is reasonable to assume that for such features the free monomer concentration is approximately the same at corresponding points in the curves for different polymer concentrations. We can then write

$$
c_{T}=a[R]+c_{f}
$$



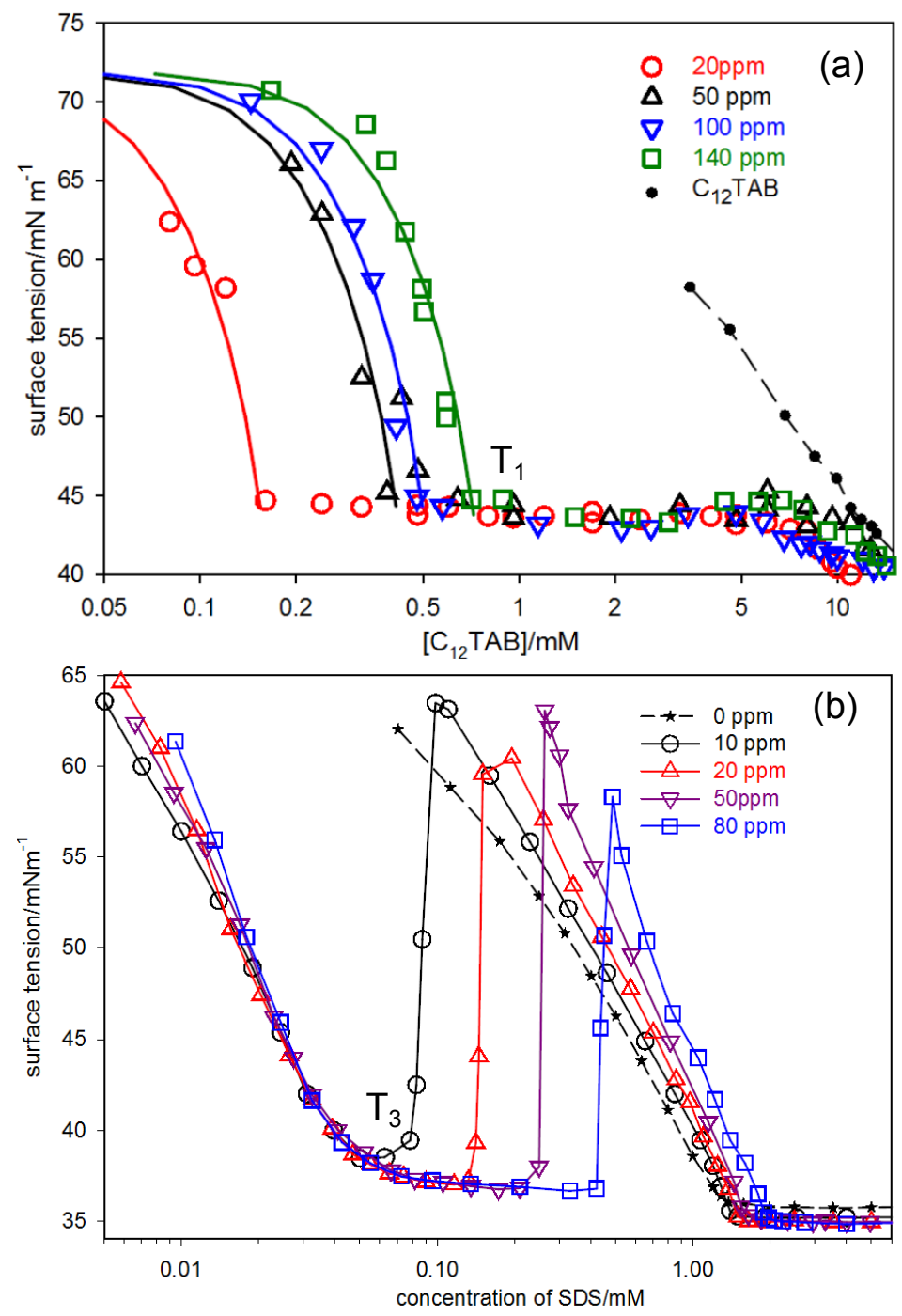

Figure 5: The surface tensions (a) NaPSS- $\mathrm{C}_{12} \mathrm{TAB}$ and (b) PDMDAAC-SDS in $0.1 \mathrm{M} \mathrm{NaCl}$ mixtures at different polymer concentrations. The data are redrawn from (a) Taylor et al ${ }^{12}$ and (b) Staples et al. ${ }^{3}$ The lines drawn through the initial drop in ST in (a) are fits to the initial ST by Bahramian et al, ${ }^{10}$ but are primarily there to guide the eye. 


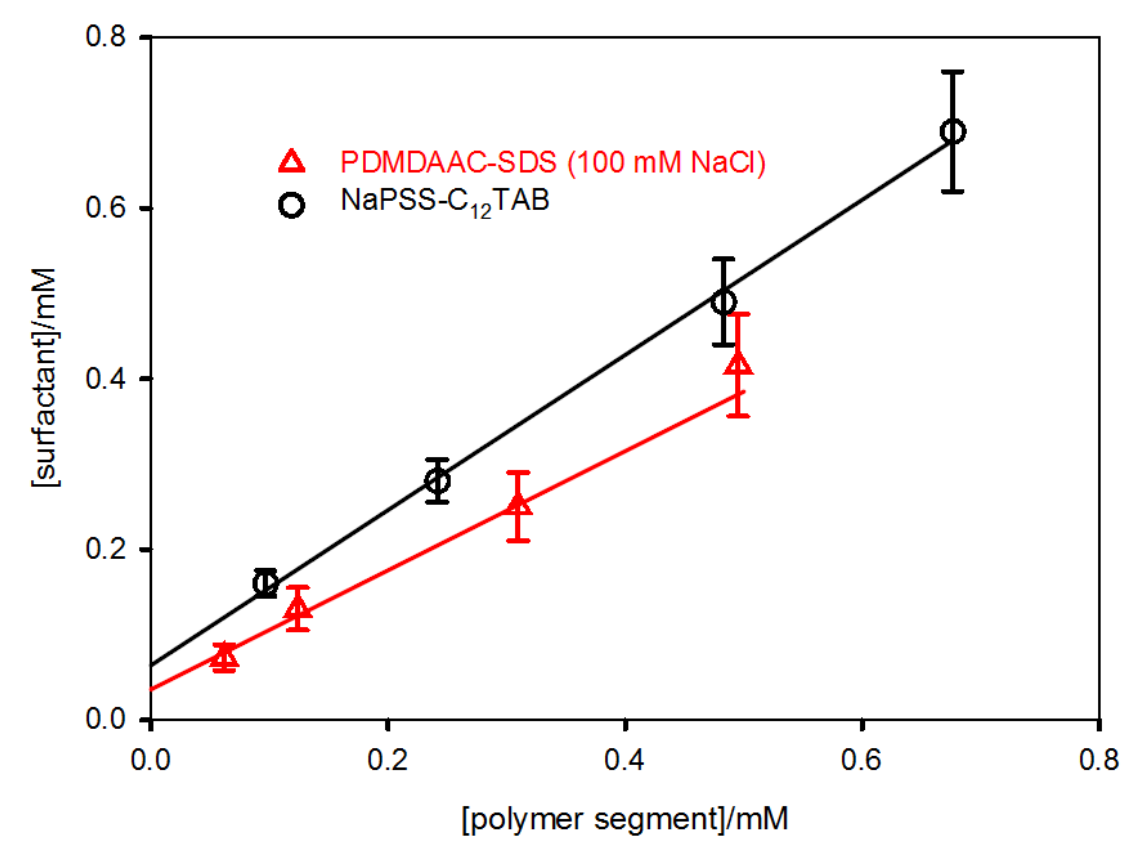

Figure 6: Determination of the free surfactant concentration using Eqn (4) and data for the variation of $\mathrm{T}_{1}$ of $\mathrm{NaPSS}_{-} \mathrm{C}_{12} \mathrm{TAB}^{12}$ and $\mathrm{T}_{3}$ of PDMDAAC-SDS $+100 \mathrm{mM} \mathrm{NaCl}^{3}$ at different polymer concentrations.

where $c_{T}$ is the concentration of any feature that is the same for solutions of different amounts of polymer, $[\mathrm{R}]$ is the polymer monomer concentration and $c_{f}$ is the free surfactant concentration. Figure 6 shows the use of this equation to determine the concentration of free surfactant at the onset of the surface plateau, $\mathrm{T}_{1}$, for NaPSS- $\mathrm{C}_{12} \mathrm{TAB}^{12}$ and at the onset of the sharp rise in $\mathrm{ST}, \mathrm{T}_{3}$, for PDMDAAC-SDS. ${ }^{3}$ The value of the free surfactant at each of these points is determined using the extrapolations shown in Figure 6 and Eqn (4), and was found to be $0.064 \pm 0.05 \mathrm{mM}$ and $0.035 \pm 0.05 \mathrm{mM}$ for NaPSS-C ${ }_{12} \mathrm{TAB}$ and PDMDAAC-SDS respectively. These values locate these particular ST features on the binding curves and they are shown as lines in Figures 2 and 3 with a shaded region representing the error.

An independent comparison of the bound fraction with the onset of the low ST plateau, which does not require the assumption above, is to use the experimental binding curve to 


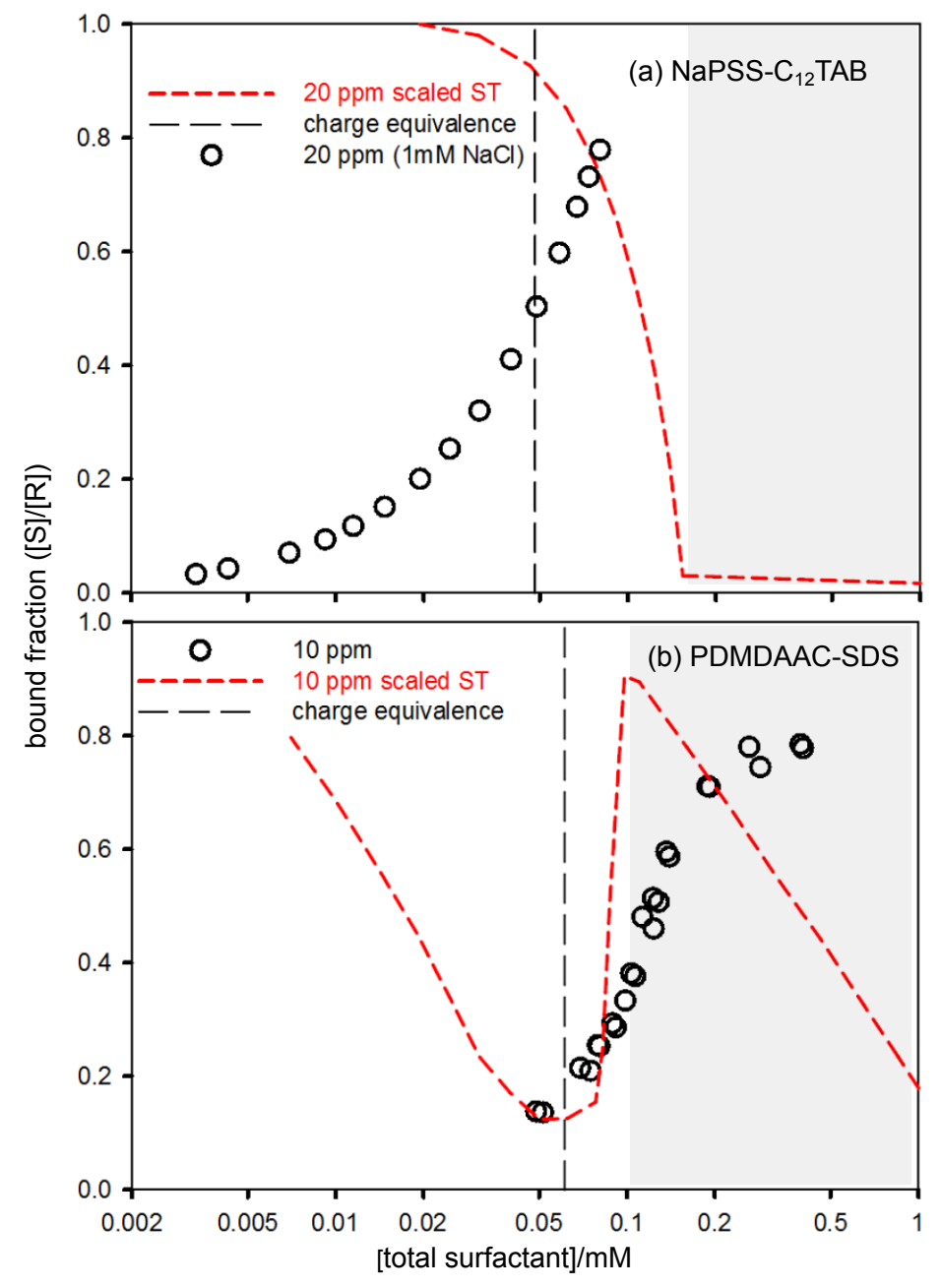

Figure 7: Experimental binding curves as a function of total surfactant concentration for (a) 20 ppm NaPSS-C 12 TAB (binding data scaled from Hayakawa and Kwak data at $21 \mathrm{mM}$ $\mathrm{NaCl}^{22}$ ) and (b) 10 ppm PMDAAC-SDS in $0.1 \mathrm{M} \mathrm{NaCl}$ (binding data from Nizri et al. ${ }^{26}$ ). The shaded regions mark part of the precipitation region as determined for PDMDAACSDS by Staples et al. ${ }^{3}$ and Varga and Campbell ${ }^{11}$ and for $\mathrm{NaPSS}_{-} \mathrm{C}_{12}$ TAB by Hansson and Almgren. ${ }^{24}$ Vertical dashed lines mark the the theoretical charge equivalence point. 
convert the bound fraction into a function of the total surfactant given by

$$
c_{\text {total }}=c_{\text {free }}+\phi \times[R]
$$

where $\phi$ is again the bound fraction and $[R]$ is the molar monomer concentration. The results can then be compared more directly to the observed ST plots and they are shown in Figure 7. The application of Eqn (5) is straightforward for PDMDAAC-SDS because the binding isotherm is independent of polymer concentration, as already discussed. It is less straightforward for NaPSS- $\mathrm{C}_{12} \mathrm{TAB}$ because the lowest concentration published data are for $100 \mathrm{ppm} \mathrm{NaPSS}$ and $21 \mathrm{mM} \mathrm{NaCl}$ and the binding curves are known to depend on both electrolyte concentration ${ }^{22}$ and polymer concentration. ${ }^{24}$ Hansson and Almgren showed that the binding constant is inversely proportional to $[\mathrm{P}]$, and Hayakawa and Kwak (HK) found a good linear $\log [\mathrm{NaCl}]-\log [1 / K]$ extrapolation to experimental data down to $6 \mathrm{mM} \mathrm{NaCl}$. We have used these two results to scale the 100 ppm $21 \mathrm{mM} \mathrm{NaCl} \mathrm{HK}$ data to 20 ppm and 1 $\mathrm{mM} \mathrm{NaCl}$ to give the plot shown in Figure 7(a). The comparison of the binding curves with the scaled ST and onset of precipitation on the same total surfactant scale confirms that the range of low ST is similar, even though the binding constants in the two systems differ by an order of magnitude. This is a result of the opposite changes in the surface activity of the two complexes. Precipitation is associated with approximate saturation of the two PE by surfactant, as would be expected. We have used the onset of precipitation obtained by Hansson and Almgren ${ }^{24}$ but we note that this has recently been challenged by Varga and Campbell ${ }^{11}$ and we discuss this disagreement further below.

The overall ST behaviour can now be summarized in terms of the binding curves. NaPSS$\mathrm{C}_{12}$ TAB shows $\mathrm{N}$-binding at average bound fractions less than about 0.1 but the low bound fraction is not enough to induce a low ST. The binding then becomes cooperative as indicated by the binding isotherms, but only over a limited range that falls well short of complete occupation of all the binding sites. This suggests that the cooperativity in this range results 
in micelles distributed relatively uniformly between the available PE molecules. Thus, either the bound fraction is again too low for surface activity or the mechanism of Eqn 3 is insufficient to make the complex surface active. The second N-binding region is reached at not a very high average bound fraction. Almgren et al. suggested that this was probably due to neutralization of polyion charges when the chain wraps around the micelles. Repulsion between partially charged micelles would then cause the chain to becomes more extended, as required for a low ST. Regardless of the mechanism of loss of cooperativity, full surface activity will not occur until the chain is both reasonably extended and almost saturated with surfactant. In PDMDAAC-SDS-100 mM NaCl C-binding dominates the initial adsorption. At the most cooperative limit, C-binding produces a small number of complexes with a high $\mathrm{S}$ content at low average bound fractions, which have the potential to lower the ST via the mechanism of Eqn 3 as described above. However, as C-binding becomes more extensive it will eventually produce 3-D complexes that are not at all surface active. As surfactant binding approaches saturation, the small number of surface active complexes of lower surfactant content that maintain the ST at a low level, are eventually lost. If the threshold concentration of surface active complexes required for a low ST is low the transition from low to high surface tension will start to occur approximately when all but the small concentration of surface active complexes required to maintain a low ST, are nearly saturated. The concentration range over which this loss of surface activity occurs will then be about the same as that required for the initial drop in ST approaching $\mathrm{T}_{1}$, which is about 0.045 $\mathrm{mM}$. The corresponding loss of surface activity then occurs from about 0.06 to just above $0.1 \mathrm{mM}$ for the $10 \mathrm{ppm}$ sample, which is easily observed on a log scale. However, at 100 ppm where the transition occurs at about $0.6 \mathrm{mM}$ the width of the transition on a log scale is reduced by $1 / 10$ th and it appears to be very sharp. This is probably an artefact of the measurements, all of which have been made in terms of $\log$ (concentration).

None of the above mentions precipitation and most of the processes above precede precipitation. In principle, precipitation is thermodynamically a separate process from the surface 
behaviour in that the activities, and hence the surface activities, of the components are not expected to change at phase separation. However, precipitation may interfere with any experimental measurement on the surface. We consider the possible effects of precipitation in the next two sections.

\section{Precipitation and Low Solubility}

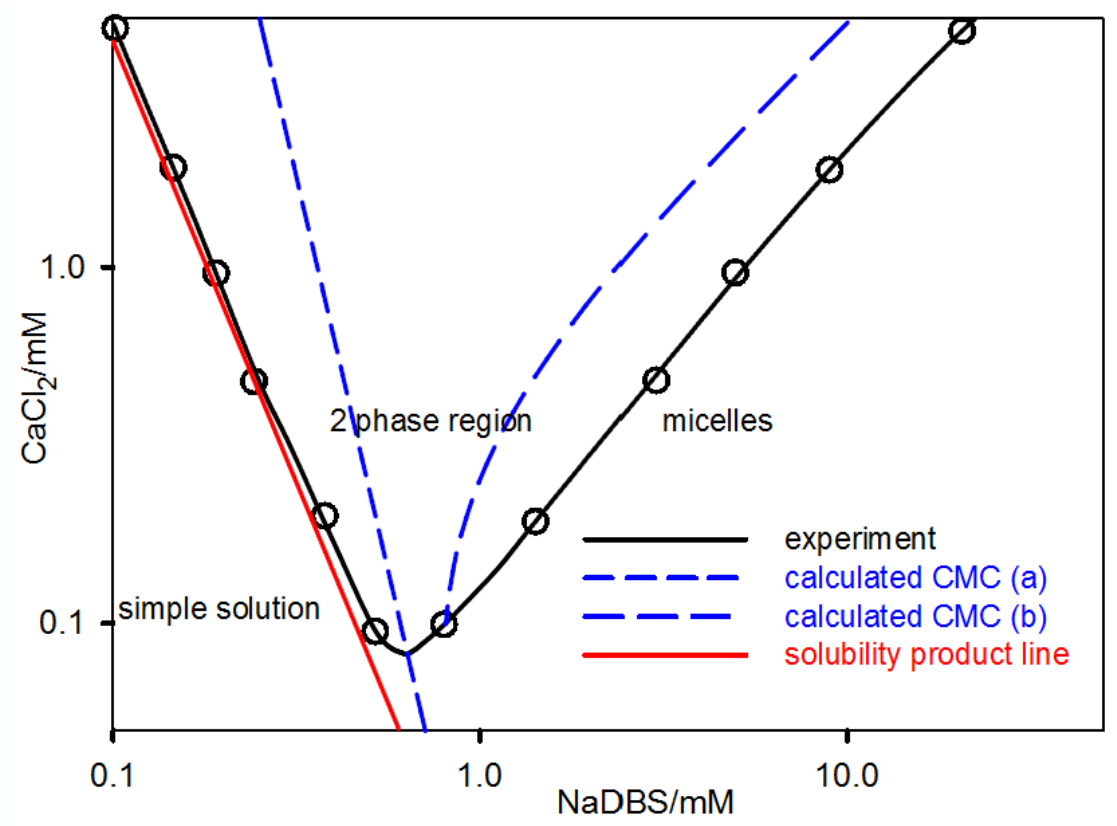

Figure 8: Solubility diagram for dilute mixtures of calcium chloride and a commercial linear sodium dodecyl benzene sulfonate, redrawn from Chou and Bae. ${ }^{31}$ The line through the experimental points is not a quantitative fit.

We have recently drawn attention to the similarity in the multilayer structures exhibited by $\mathrm{PE}-\mathrm{S}$ and $\mathrm{M}^{n}-\mathrm{S}$ systems, ${ }^{13}$ which suggests that studies of the simpler $\mathrm{M}^{n}-\mathrm{S}$ systems might give useful insight into the layering in PE-S systems. The basic solubility pattern for both systems can be represented by the example of the experimental phase diagram for the addition of $\mathrm{CaCl}_{2}$ to a commercial linear sodium dodecyl benzene sulfonate surfactant (NaDBS) in aqueous solution, ${ }^{31}$ which is shown in Figure 8. The solubility boundary is the line on the left hand side of the Figure and is determined by the value of the solubility product. Addition of surfactant to the right of the solubility line initially causes further 
precipitation of $\mathrm{M}^{n} \mathrm{~S}_{n}$, but when its CMC is reached $\mathrm{M}^{n}$ ions will start to be incorporated into the $\mathrm{M}^{1} \mathrm{~S}$ micelles. To maintain equilibrium then requires redissolution of some of the $\mathrm{M}^{n} \mathrm{~S}_{n}$ precipitate. It is not easy to determine the onset of redissolution except that it must lie between a lower limit equal to the mixed CMC and a higher limit equal to the amount of surfactant required to precipitate all the $\mathrm{M}^{n}$ present as $\mathrm{M}^{n} \mathrm{~S}_{n}$. These limits are represented by dashed lines in the Figure. The precipitate continues to be solubilized until the system becomes a micellar phase at the redissolution line on the right of the Figure. Although the phase diagram of Figure 8 gives little information about the mechanism of the changeover between precipitation and redissolution, the region between the precipitation and redissolution curves is clearly a two phase region where the activity of the precipitated $\mathrm{M}^{n} \mathrm{~S}_{n}$, and hence the solution activity, is approximately constant. The redissolution line is an approximate measure of the solubilization ratio (effectively the ratio of the total concentration of $\mathrm{M}^{1} \mathrm{~S}$ to the initial concentration of $\mathrm{M}^{n} \mathrm{X}_{n}$ ). There may also be micelles present in part of the two phase region and they are present in the final solution. The line through the experimental points of the redissolution line in Figure 8 is just a guide to the eye but, for the simple case described and in dilute conditions, both arms of the plot can be calculated when activity coefficients, values of the association constants of the two ions to the micelles and the precipitate, and the effect of the added electrolyte on the CMC are included. ${ }^{4,31-35}$ When further surfactant is added beyond complete redissolution the $\mathrm{M}^{n}$ ion continues to be solubilized into the micelles, which reduces its activity in the solution until the micelles are saturated. There are a number of further possible complications in $\mathrm{M}^{n}-\mathrm{S}$ systems but for our present purposes most of these are unimportant. However, we mention that the same failure to reach equilibrium by the formation of a colloidal suspension as is commonly found in PE-S systems sometimes occurs. This manifests itself experimentally as a horizontal line intersecting the redissolution line at a threshold $\mathrm{M}^{n} \mathrm{X}_{n}$ concentration. ${ }^{4}$

It is relatively easy to assess the ST behaviour corresponding to a typical measurement along a horizontal cut through the phase diagram. First, since surfactants with multivalent 
ions are more surface active than those with monovalent ions, the initial addition of surfactant lowers the ST progressively and this part should be quantitatively explained by the Gibbs equation. Secondly, at concentrations of S above the precipitation line the bulk concentration and hence the ST of the surface active component $\mathrm{M}^{n} \mathrm{~S}_{n}$ will be approximately constant. In practice, since the activity coefficients change on further addition of surfactant the ST may slightly increase or decrease slightly in this region. For a nonionic compound, the slope of the $\mathrm{ST}-\ln [\mathrm{S}]$ plot can change at the point of precipitation but there is no step change in the ST. This is because, the chemical potentials for the solute are the same in the precipitate, the saturated solution and the surface layer, ${ }^{36}$ i.e.

$$
\mu_{S}^{\prime}=\mu_{S}^{\prime \prime}=\mu_{S}^{\gamma}
$$

where $\mu_{S}^{\prime}, \mu_{S}^{\prime \prime}$ and $\mu_{S}^{\gamma}$ denote the chemical potentials of nonionic surfactant $\mathrm{S}$ in the bounding bulk phases (' and ") and the interfacial region $\left({ }^{\gamma}\right)$. Note that $\mu^{\gamma}$ here is as defined in the Gibbs convention, for which Eqn (6) holds for a plane surface at equilibrium. The relation does not hold for non-planar interfaces where $\mu^{\gamma}$ then partly depends on the chemical potentials of the bounding phases. ${ }^{37}$ Since the Gibbs equation at constant temperature is

$$
d \gamma=-\Gamma_{S} d \mu_{S}
$$

where $\Gamma_{S}$ is the surface excess of $S$, Eqn (6) requires there to be no step change in the ST at a phase transition for a nonionic surfactant. The same set of equations will hold to a good approximation for an $\mathrm{M}^{n}-\mathrm{S}$ system but the equilibrium between precipitate and solution now requires the mean activity of the components of the precipitate not to change across the phase transition. If the solution consists of pure $\mathrm{M}^{n} \mathrm{~S}_{n}$ the same mean activity applies to the adsorbed layer and Eqns (6) and (7) again hold. This will generally be a good approximation for $\mathrm{M}^{n}-\mathrm{S}$ systems.

Two examples of the ST behaviour are shown in Figure 9 for $\mathrm{AlCl}_{3}$ with (a) sodium 

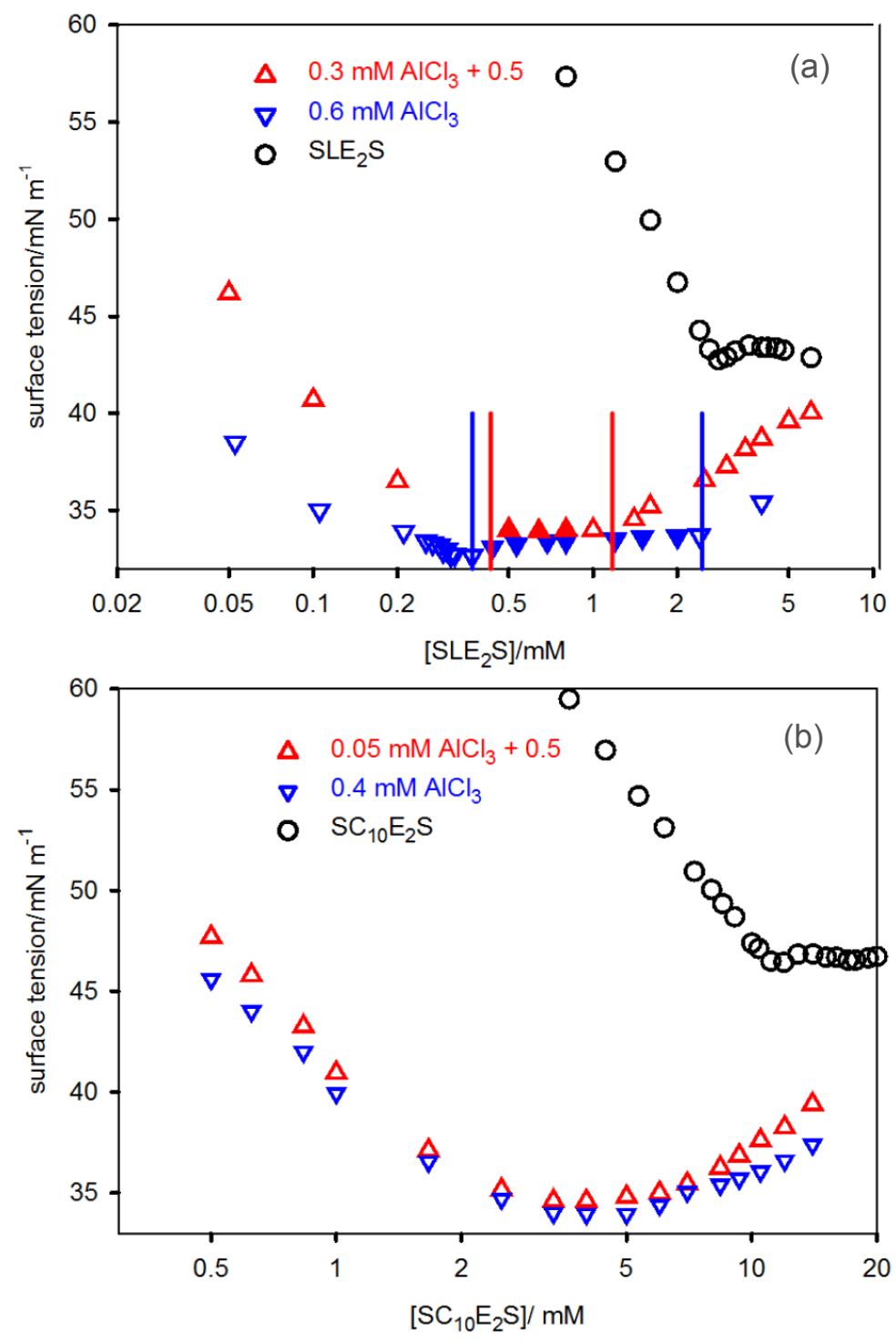

Figure 9: The variation of the surface tension of (a) $\mathrm{SLE}_{2} \mathrm{~S}\left(\mathrm{SC}_{12} \mathrm{E}_{2} \mathrm{~S}\right)$ and (b) $\mathrm{SC}_{10} \mathrm{E}_{2} \mathrm{~S}$ as the concentration of sodium surfactant is varied at different fixed $\mathrm{AlCl}_{3}$ concentrations. The vertical lines in (a) mark the boundaries of the two phase region defined in Figure 8. The filled in points in (a) indicate solutions that are visibly turbid. Data redrawn from Xu et al. ${ }^{1}$ 
dodecyldiethyleneglycol sulphate $\left(\mathrm{SC}_{12} \mathrm{E}_{2} \mathrm{~S}\right)$, where there is phase separation, and with (b) $\mathrm{SC}_{10} \mathrm{E}_{2} \mathrm{~S}$, where there is no phase separation at comparable $\mathrm{AlCl}_{3}$ concentrations. ${ }^{1}$ That the two breaks at either end of the approximate plateau in (a) are associated with phase separation is confirmed approximately by the visual turbidity. There is no step change in the ST at phase separation at either break, as expected, there is a slight upward slope in the ST plateau, and the greater width of the plateau indicates that the ST is slightly more sensitive than turbidity measurements to the phase separation. The first break is the onset of precipitation and the second is the completion of redissolution. There is no turbidity and no indication of an $\mathrm{ST}$ plateau for the more soluble $\mathrm{SC}_{10} \mathrm{E}_{2} \mathrm{~S}$, which is consistent with no phase separation, although precipitation and the plateau do occur at higher starting concentrations of $\mathrm{AlCl}_{3}$. Small angle neutron scattering (SANS) also confirms that both these systems are micellar at higher surfactant concentrations, ${ }^{15}$ i.e. they do not form non-equilibrium colloidal dispersions in the redissolved region. A striking feature of this comparison is that the overall magnitude and direction of the ST changes is the same whether or not there is precipitation. The phase change does not alter the underlying ST behaviour except that smooth changes become sharp ones at the two phase boundaries.

The precipitation of a simple non-ionic solid would give a horizontal plateau above the first precipitation step because the system is invariant. Thus the phase rule for a system containing only plane surfaces is ${ }^{37}$

$$
w=2+(c-r)-p-(\phi-s)
$$

where $w$ is the number of degrees of freedom, $c$ the number of components connected by $r$ chemical reactions, $p$ the number of bulk phases, $\phi$ the number of surface phases and $s$ the number of distinct surfaces. This becomes the same as the normal phase rule when there is only one surface phase per surface. For a non-ionic solid at saturation in water with a nitrogen atmosphere, i.e. 3 components and 3 phases, $w=2$ and if $T$ and $P$ are fixed the 
system is invariant, i.e. the concentration and hence the ST are fixed. In $\mathrm{M}^{n}-\mathrm{S}$ systems + nitrogen there are 5 independent components. In the plateau region $w$ is therefore 4 and, since $T, P$ and the $\mathrm{M}^{n}$ concentration are fixed the system is univariant, i.e. the ST can vary with surfactant concentration and is not restricted to a plateau. In the $\mathrm{AlCl}_{3}-\mathrm{SC}_{12} \mathrm{E}_{2} \mathrm{~S}$ system the solubility of $\mathrm{AlS}_{3}$ will be relatively insensitive to added surfactant and the ST is therefore close to a horizontal plateau.

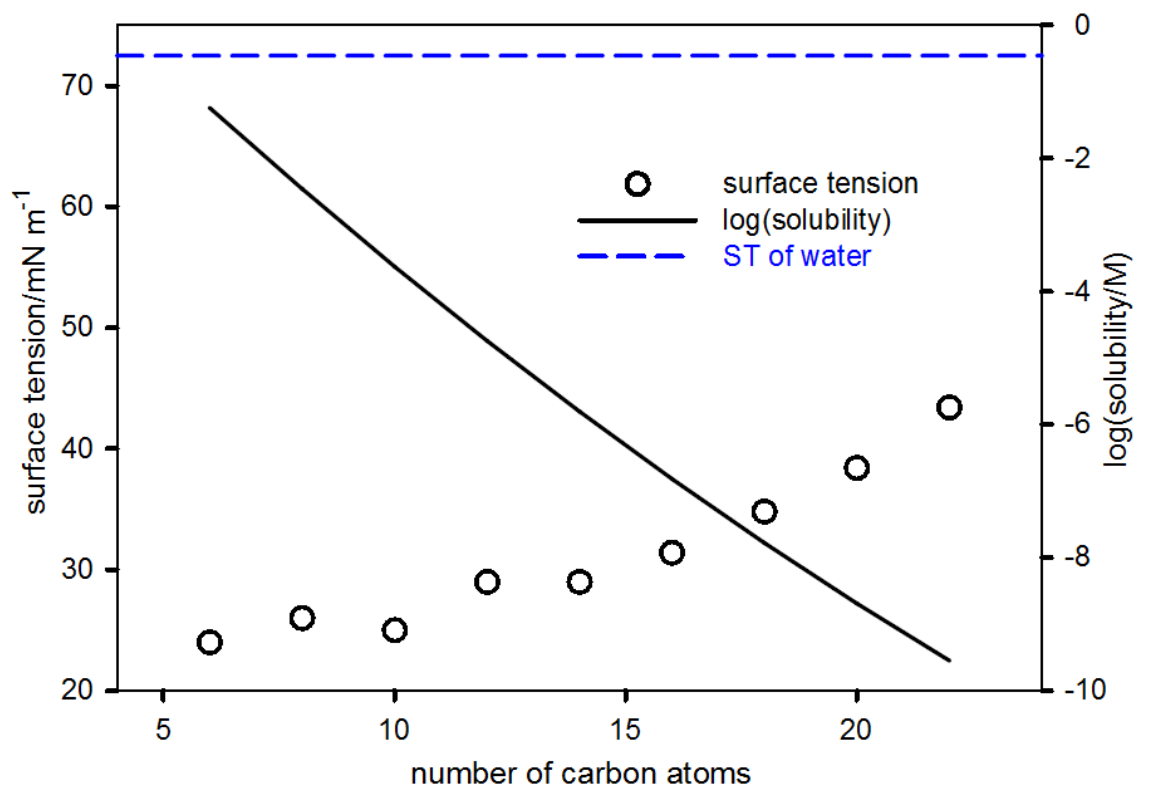

Figure 10: Limiting surface tension at saturation (or equilibrium spreading pressure) and bulk solubilities of $n$-alkanols. The ST data are taken from from Deo et al. ${ }^{38}$ and Prigogine and Defay ${ }^{37}$ and the solubility is based on an empirical equation. ${ }^{39}$ The ST of water is shown for comparison as a dashed line

$\mathrm{PE}-\mathrm{S}$ systems differ from $\mathrm{M}^{n}-\mathrm{S}$ systems in that the precipitate is much less soluble when it occurs and because the equilibria are more complicated. Both have potentially important consequences. We address the low solubility first. At charge equivalence a PE-S system stoichiometrically is a mixture of the neutral polymer salt, which we write as PS, and electrolyte MX, and Piculell has shown that several of these salts are virtually insoluble. ${ }^{40}$ The way surface activity and low solubility interact is well illustrated by the extensive work that has been done on low solubility systems, particularly on the longer chain $n$-alkanols. 
The alkanols are surface active and their adsorption results in an ST that increases slowly with chainlength, while their solubilities simultaneously rapidly decrease. The combination of these two effects leads to the ST of their saturated solutions remaining reasonably low regardless of chainlength, as can be seen in Figure 10 where they are plotted against the log of the limiting solubility in water for $\mathrm{C}_{6}$ to $\mathrm{C}_{22}$. Thus the compounds remain moderately surface active up to and including the $\mathrm{C}_{22}$ alkanol, whose solubility is $2.8 \times 10^{-10} \mathrm{M}$ (extrapolated value $^{39}$ ). The thermodynamic definition of the solubility of a solid is via its solubility or solubility product. This is never zero and for substances that are amphiphilic the decrease in solubility with increasing chainlength often has little effect on their surface activity at saturation, as shown by Figure 10. Low solubility on its own does not affect the limiting ST at saturation.

The low solubilities of the higher alkanols do, however, lead to substantial difficulties with surface equilibration, mainly associated with depletion. The problems in the case of hexadecanol have been examined systematically by Park et al. ${ }^{41}$ They used a trough and Wilhelmy plate to measure the equilibrium spreading pressure (ESP) for spread monolayers, and both bubble and pendant drop tensiometers to measure the limiting ST of layers formed by diffusion upwards and downwards respectively, and to study the rate and mechanism of equilibration with the surface. They used suspensions of hexadecanol of varying particle size and compared these with a sample filtered with a $0.02 \mu \mathrm{m}$ pore membrane. The definitive experimental determination of the ST was made via spreading of a monolayer and determination of the equilibrium spreading pressure (ESP), using $\mathrm{ST}=\mathrm{ST}_{\text {water }}-\mathrm{ESP}$, which has long been known to be the only reliable method for sparingly soluble componds. ${ }^{42,43}$ The ST results on the diffusing systems could be well modelled in terms of a homogeneous distribution of particles acting as the primary sources of monomer adsorbate in a situation characterized by the depth of saturated solution required to generate a complete monolayer and the thickness of suspension required to generate a complete monolayer. The parameters required for the fit showed that an equilibrium ST (by direct measurement) could not be 
achieved in the absence of a dispersion of solid particles. They also observed that gravity increased the rate of equilibration in the bubble measurements but hindered it in the pendant drop, which was attributed to the effects of gravity on the hexadecanol particles with hexadecanol being less dense than water.

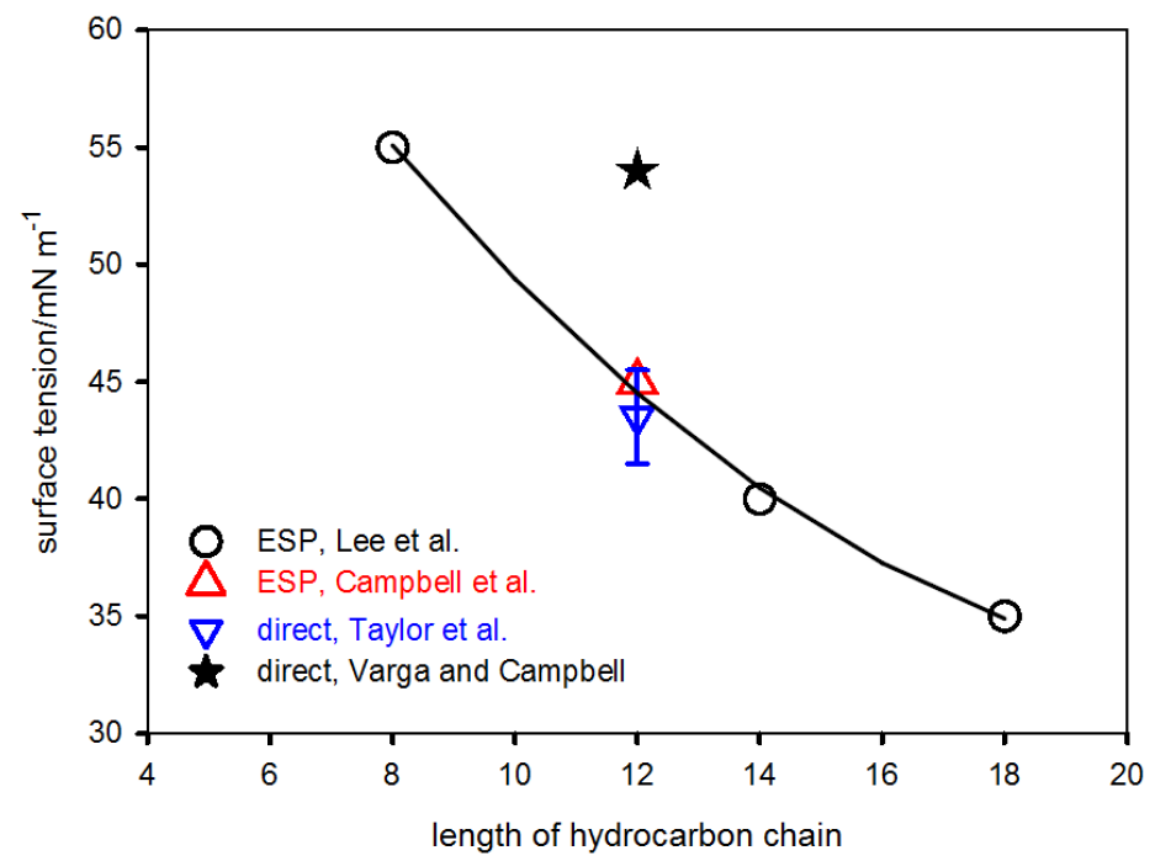

Figure 11: Values of the ST obtained from approximate equilibrium spreading pressures (ESP) for NaPSS- $\mathrm{C}_{n} \mathrm{TAB}$ mixtures and a direct measurement of the plateau ST for NaPSS$\mathrm{C}_{12}$ TAB. ESP data are from Lee et al. ${ }^{44}$ and Campbell et al. ${ }^{45}\left(\mathrm{C}_{12} \mathrm{TAB}\right)$. Polymer ST data are from Varga and Campbell ${ }^{11}$ and Taylor et al. ${ }^{12}$

The alkanols demonstrate clearly that there are likely to be serious difficulties in measuring the true ST for PE-S systems when precipitation is extensive. The determination of the limiting ST using spreading methods has not been used explicitly for PE-S systems. However, two spreading experiments have been performed on NaPSS- $\mathrm{C}_{n}$ TAB systems and give interesting and relevant results. Lee et al. measured the spreading of monolayers of the precipitated complex from NaPSS- $\mathrm{C}_{8} \mathrm{TAB}$, NaPSS- $\mathrm{C}_{14} \mathrm{TAB}$ and NaPSS- $\mathrm{C}_{18} \mathrm{TAB}$. All three spread spontaneously on water starting with complexes of composition $\mathrm{RS}_{5}$. Excess surfactant dissolved in the subphase to give a final surface composition of $\mathrm{RS}_{1.2},{ }^{44}$ measured $e x$ situ. Campbell et al. later made a similar study of NaPSS- $\mathrm{C}_{12} \mathrm{TAB}$. Here the initial complex 
was precipitate taken from concentrated residue slightly above charge neutral precipitation and in situ NR showed that the spreading complex was $\mathrm{RS}_{1} \cdot{ }^{45}$ The approximate equilibrium spreading pressures (ESP) of these PE-S complexes with different surfactant chainlengths show that they are all surface active, as can be seen in Figure 11, where they are plotted as the ST. The collapse pressure was measured rather than the ESP but, as discussed by Smith and Berg, ${ }^{46}$ the onset of collapse of a spread monolayer under pressure is a good approximation for the ESP. Also included in the diagram is the average ST of the long plateau for NaPSS- $\mathrm{C}_{12}$ TAB measured directly by Taylor et al., ${ }^{12}$ which is in good agreement with the ST from the ESP of the complex used by Campbell et al. and with the trend in Figure 11. However, the ESP of the complex used by Campbell et al. disagrees with Varga and Campbell's own direct measurement of $54 \mathrm{mN} \mathrm{m}^{-1}$, which is also plotted in the figure. We discuss this result further below.

The other important feature to consider is how the thermodynamic restrictions of Eqn (6) are relaxed by the more complex equilibria in a $\mathrm{PE}-\mathrm{S}$ system. The equilibria at precipitation can be represented by

$$
\begin{gathered}
\mathrm{RS}_{m} \mathrm{X}_{1-m} \leftrightarrows \mathrm{R}+m \mathrm{~S}+(1-m) \mathrm{X} \\
\mathrm{RS}_{n} \leftrightarrows \mathrm{R}+n \mathrm{~S}
\end{gathered}
$$

where $m$ and $n$ are the stoichiometries of the adsorbed layer and the bulk solution/precipitated complex respectively. The mean activity of the precipitated species must be the same in solution and precipitate at the phase transition but if $m \neq n$ the mean activity of the adsorbed layer may change at the transition. In general, the stoichiometry of the precipitate is close to 1 and that of the adsorbed layer slightly lower than 1 and the effect should therefore be small. The conditions of Eqn (6) and (7) clearly hold closely for the example given in Figure 9 but more care may be needed in applying them to PE-S systems. In the positions drawn for precipitation in Figure 7 there are no step changes in the ST, as expected from the above argument, although there are large changes in the slopes on either side. However, 
the positions of the phase changes may not be sufficiently accurately known to make the comparison and, as already mentioned, there is disagreement about the phase change in NaPSS- $\mathrm{C}_{12} \mathrm{TAB}$, which is discussed in a later section.

\section{Equilibration}

There are two mechanisms that may prevent equilibrium being established at the surface of PE-S systems. One is the slow chain dynamics of the polymer chain and the other is that precipitation may lead to a colloid. There has been little exploration of the first in $\mathrm{PE}-$ S systems but considerable attention has been given to the second, which also occurs less frequently in $\mathrm{M}^{n}-\mathrm{S}$ systems. The presence of colloidal particles must in principle interfere with equilibration at the surface because colloids are inherently non-equilibrium particles. The concept of using equilibrium principles to discuss the surface of a colloidal solution raises some interesting questions that do not seem to have been addressed.

Colloidal particles are not surface active unless there is some asymmetry in their charge or hydrophobicity and they therefore do not generally adsorb at the air-water interface. They also diffuse very slowly in comparison with molecular species and, even if they are weakly attracted to the surface, do not compete for adsorption with surface active molecular species. For the purposes of the following discussion, we assume that both these conditions hold and that colloidal particles of complex precipitate are formed on mixing. In the two phase region what is required for solution and surface equilibrium is the presence of a sufficient fraction of normal precipitate to ensure that the solution is saturated. Since the colloid is generated by random mixing and the solubility is low enough for little normal precipitate to be required for saturation, it would be unusual for the solution not to be saturated, and hence true surface equilibrium achieved. In the one phase region the solution may contain some colloidal particles as a result of inhomogeneous mixing. Depending on the stoichiometry of the particles the solution will gradually change to a new and unknown composition as the particles dissolve. Because the precipitate will be not be very different 
from the stoichiometry of the normal complex the result will be a solution with a slightly different S-PE ratio, i.e. the surface will be slightly different from the true equilibrium expected for the nominal composition. Unless the majority of the complex is in colloidal form, neither of these situations will interfere significantly with NR or ST measurements except to introduce some additional inaccuracy, i.e. these measurements are not strongly affected. However, the bulk physical properties may be more strongly affected depending on how they respond to colloidal particles. For example, turbidity measurements might be strongly affected.

A defining feature of colloidal particles is that they are significantly less than $1 \mu \mathrm{m}$ in diameter. A colloid therefore has a high internal area. For example, the colloidal particles in $1 \mathrm{~L}$ of solution containing $100 \mathrm{ppm}$ NaPSS and forming a nearly complete precipitate with $\mathrm{C}_{12} \mathrm{TAB}$ of stoichiometry about RS have a total mass of about $0.25 \mathrm{~g}$. For spherical particles at the maximum size to count as colloidal, i.e. a diameter of $1 \mu \mathrm{m}$, the resulting surface area is $1.5 \mathrm{~m}^{2}$. However, given the rapid formation of these particles and the involvement of polymers the diameters are more likely to be in the hundreds of $\AA$ with non-spherical shapes, which could further increase the area. For $200 \AA$ spherical particles, for example, the total internal surface area of the same amount of sample increases to $75 \mathrm{~m}^{2}$ (the internal area scales as $1 / r)$. The surface active species maintaining the low $\mathrm{ST}$ is typically also of stoichiometry RS and would probably adsorb as a monolayer with an area per unit of about $50 \AA$. The complete coverage of the colloidal surface would then require $2.5 \times 10^{-4}$ moles of RS or half the total amount of RS in the system. If we assume that the colloid is only formed from $50 \%$ of the initial material half the RS becomes colloidal particles and half of the remainder is lost in adsorption on the colloidal surface, leaving a final solution concentration $25 \%$ of the original. This is a metastable equilibrium but it is not the full equilibrium appropriate to the nominal concentration and there is the more serious possibility that one component is preferentially adsorbed. Although the above is an approximate calculation and the system could, to some extent, readjust, it indicates that depletion effects in these system must be 
taken seriously. Interestingly, the reverse problem occurs in the determination of adsorption isotherms at the solid-liquid interfaces, in which depletion of the solute is the method of choice for measurement and the objective is then to make the surface area of the adsorbent as high as possible. For example, Gispert et al. used alumina powder with a total surface area of $3 \mathrm{~m}^{2}$ in $10 \mathrm{~mL}$ of a protein solution to study the adsorption of serum albumin, a similar condition to that generated by a $\mathrm{PE}-\mathrm{S}$ system in the two phase region, but designed to obtain a significant adsorbed fraction. ${ }^{47}$

Colloidal precipitation in any system will nearly always result in a high area non-equilibrium surface. If this surface also adsorbs, it will compete with the air-water interface. In PE-S systems, since adsorption of a complex is likely to be comparably strong at air-water and at colloid-water interfaces and, since the area of the colloidal surface is orders of magnitude greater than the geometrically defined air-water interface, adsorption at the colloidsolution interface can potentially overwhelm any air-water measurement. The effect cannot be avoided by changing the sample size because it is an internal surface and is not affected by sample size. However it is dependent on the extent of precipitation and should therefore be small except in the two phase region, where it will reach a maximum when precipitation is a maximum. Colloidal dispersions are often metastable and are vulnerable to mechanical disturbance because it brings non-equilibrium particles into contact either with each other or with containing surfaces. For example, Marangoni effects would surely break a PE-S colloidal particle into its constituent fragments upon any collision of such a particle with any interface. The conclusion is that the more a two phase PE-S sample is disturbed the closer it, and especially its air-water interface, will be to equilibrium. The corollary is that with minimum disturbance the air-water interface is more likely to be prevented from reaching equilibrium by competitive adsorption at the colloid-water interface. 


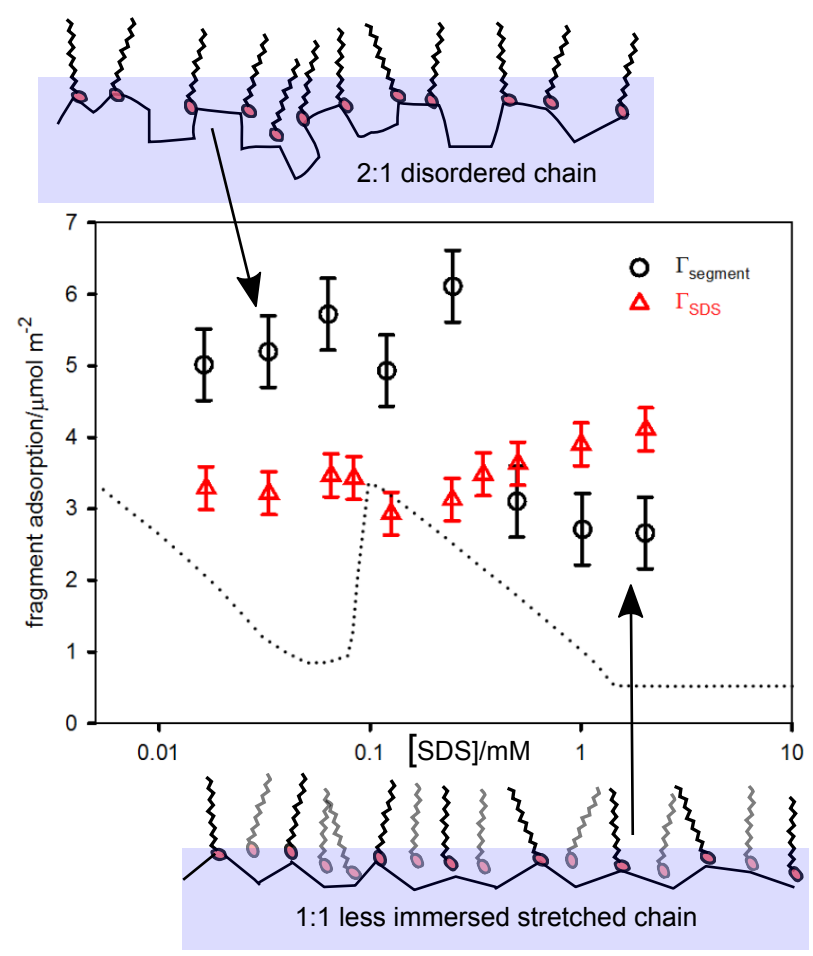

Figure 12: Values of the adsorbed amounts of SDS and DMDAAC segments determined by $\mathrm{NR}$ at a PDMDAAC concentration of $10 \mathrm{ppm}$. The segment surface excesses are calculated from the volume fraction and thickness of polymer in the adsorbed layer given by Staples et al., ${ }^{3}$ using an segment volume of $260 \AA^{3}$ estimated from the bulk density of PDMDAAC. The ST behaviour (dotted line) is shown on an arbitrary scale for comparison.

\section{Mixing and Surface Tension}

In a $\mathrm{PE}-\mathrm{S}$ system with only a single surfactant the issue of surface mixing arises if there is a crossing point of the ST curves of two surface active groups of species, usually a PE-S complex and the surfactant at higher concentration. The surface phases of each of these states are expected to be miscible since they are relatively similar. The ST of the surfactant at high concentration will generally be close to that of the free surfactant but, in some cases there is polymer adsorption and this will lead to a small additional reduction in the ST. This can be observed either indirectly from the ST at high surfactant concentrations or directly with NR. For example, for PDMDAAC-SDS (100 mM NaCl) Figure 5(b) shows that above the CMC of SDS the ST in the presence of polymer decreases by about $1 \mathrm{mN} \mathrm{m}^{-1}$, which by the Gibbs equation means that some polymer is adsorbed. This is supported in more 
detail by NR results. Figure 12 illustrates the change. The surface excess of polymer can be determined by matching the SDS to the solvent, i.e. making them both deuterated, so that the reflected signal is entirely from the polymer at the surface. Although the accuracy is lower than that with which the surface excess of SDS can be determined, it is sufficient to show what is occurring in this system. The adsorbed complex below $\mathrm{T}_{3}$ has the approximate stoichiometry $\mathrm{R}_{2} \mathrm{~S}$, indicating that only about half the segment charges can be neutralized by surfactant, as shown schematically at the top of Figure 12. There is a dip in the coverage of both polymer and surfactant at the ST peak, but then the higher concentration of surfactant lowers the free energy of the normal free surfactant surface so that it can form a more stable mixed monolayer with the $\mathrm{R}_{2} \mathrm{~S}$. The decrease brought about by the extra favourable free energy of mixing of two systems of approximately equal surface activity then creates a stable layer with equal amounts of each component, i.e. a 1:1 or $R_{1} S_{1}$ layer in total, as observed by NR. This presence of polymer below, at, and above the ST peak was the key result that caused Staples et al. to argue that depletion by precipitation was not responsible for the presence of the ST peak. This measurement also directly contradicts the conclusion of Varga and Campbell that all the polymer is lost by precipitation, which we discuss further below.

The more interesting type of mixing in these systems occurs when surfactant mixtures are used to change the mean properties of the surfactant. Thus mixtures of charged and uncharged surfactant can be used to change the cooperative component of the binding and hence to tune the ST behaviour. Anionic SDS and the nonionic $\mathrm{C}_{12} \mathrm{E}_{6}$ interact synergistically and the ST of this mixture with PDMDAAC and PEI has been studied in some detail. ${ }^{48-50}$ The synergy between the two surfactants is partly distorted by the large difference in the CMCs of the two surfactants, $8 \mathrm{mM}$ for SDS and $0.07 \mathrm{mM}$ for $\mathrm{C}_{12} \mathrm{E}_{6}$, but these change to $1.3 \mathrm{mM}$ and $0.07 \mathrm{mM}$ in $100 \mathrm{mM} \mathrm{NaCl}$.

A selection of features in the $\mathrm{ST}$ of PDMDAAC-SDS- $\mathrm{C}_{12} \mathrm{E}_{6}$ systems in $100 \mathrm{mM} \mathrm{NaCl}$ is shown in Figure 13. ${ }^{51}$ Figure 13 (a) shows the results for a fixed PDMDAAC composition 


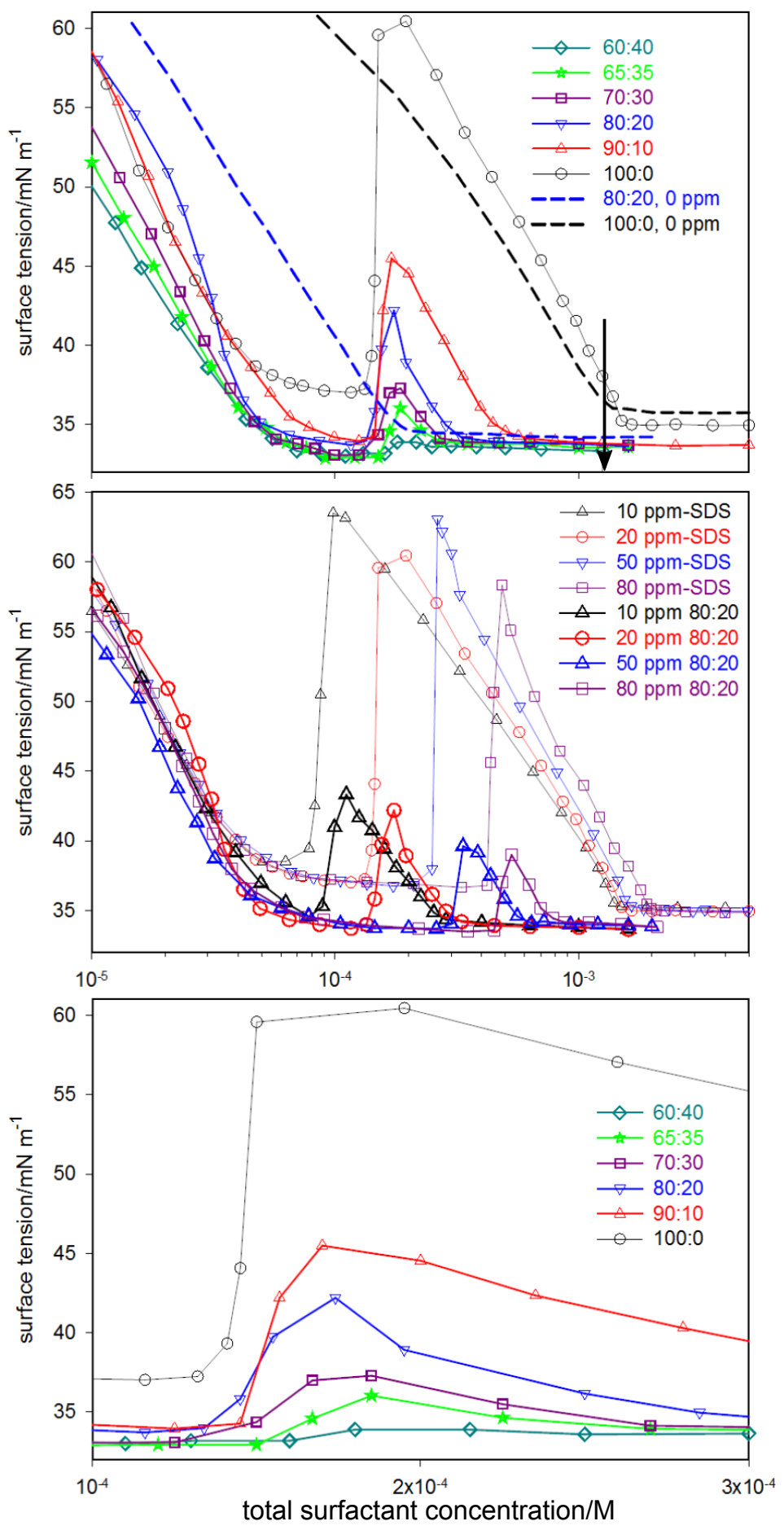

Figure 13: Surface tension of PDMDAAC-SDS- $\mathrm{C}_{12} \mathrm{E}_{6}-100 \mathrm{mM} \mathrm{NaCl}$ mixtures (a) $20 \mathrm{ppm}$ PDMDAAC and different surfactant compositions (dashed lines show the surfactant in the absence of PDMDAAC at 100\% SDS and 80\$ SDS, (b) 10, 20, 50 and 80 ppm PDMDAAC and a constant ratio of 80:20 SDS: $\mathrm{C}_{12} \mathrm{E}_{6}$, (c) the same as (a) but over a limited linear concentration range. Data redrawn from Staples et al. ${ }^{51}$ 
of $20 \mathrm{ppm}$ and a sequence of $\mathrm{SDS}: \mathrm{C}_{12} \mathrm{E}_{6}$ ratios, Figure 13(b) shows the results for four PDMDAAC concentrations with pure SDS and with 80:20 SDS:C $\mathrm{C}_{12} \mathrm{E}_{6}$, and Figure 13(c) replots the results from (a) on a linear concentration scale. Although complicated by the extra variable of the surfactant composition, the results follow a pattern of peak position and intensity that seems too systematic for anything other than equilibrium behaviour. In this context, it is interesting that, although this is the largest set of ST peaks reported in the literature, it has not yet been included as part of any discussion that couples ST peaks with the question of equilibration.

There are a number of interesting features in Figure 13, (i) the ST peaks are substantially reduced by the introduction of a relatively small proportion of nonionic surfactant, (ii) the peaks are displaced towards higher total surfactant concentration with increasing $\mathrm{C}_{12} \mathrm{E}_{6}$, (iii) the ST has a stronger polymer concentration dependence below $\mathrm{T}_{1}$ than for $100 \%$ SDS, (iv) the limiting ST of the mixtures at $\mathrm{T}_{3}$ is lower by about $4 \mathrm{mN} \mathrm{m}^{-1}$ compared with $100 \%$ SDS, and (v) the linear plots emphasize that the apparent sharpness of the ST peaks results in part from the use of a $\log$ (total surfactant) scale. Features (i), (ii) and (iii) are consistent with an enhancement of the stability of adsorbed complexes by $\mathrm{C}_{12} \mathrm{E}_{6}$. The presence of uncharged surfactant molecules in the outer part of the layer would allow a more relaxed chain conformation and hence a more stable layer in the region where the complex is surface active. As C-binding becomes more dominant with higher fractional binding it is less obvious what the effect of the nonionic surfactant might be. The calculations below suggest that nonionic surfactant is increasingly squeezed out of the complex and hence has little impact on its surface activity. However, the greater surface activity of the mixture means that the ST is dominated by free surfactant at lower bulk concentrations.

The measurements of composition by NR on the same system are shown in Figure 14 and have a further set of interesting features, (i) the total adsorption (not shown) below $\mathrm{T}_{1}$ is enhanced by about $20 \%$ when $\mathrm{C}_{12} \mathrm{E}_{6}$ is present, (ii) at the lowest measurement below $\mathrm{T}_{1}$ about $30 \%$ of the layer for the $80: 20$ composition is nonionic surfactant, (iii) the fraction of 


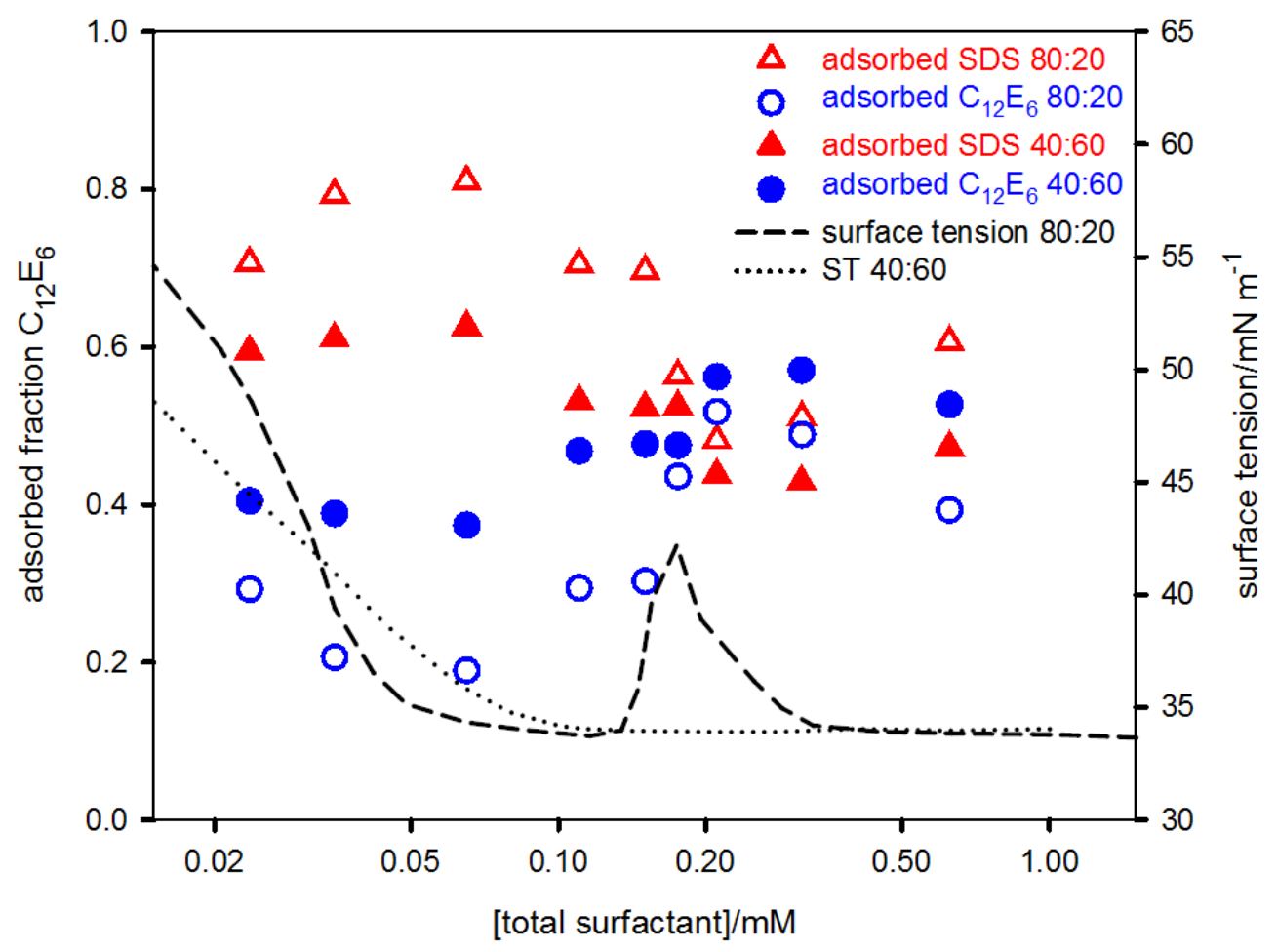

Figure 14: Adsorbed fractions of SDS and $\mathrm{C}_{12} \mathrm{E}_{6}$, as determined by NR, for 20 ppm PDMDAAC with different total concentrations of SDS and $\mathrm{C}_{12} \mathrm{E}_{6}$ and with SDS: $\mathrm{C}_{12} \mathrm{E}_{6}$ ratios of 80:20 and 40:60, all in $100 \mathrm{mM} \mathrm{NaCl}$. The ST variation is shown for 20 ppm PDMDAAC for each of the two compositions. Data replotted from Staples et al. ${ }^{51}$

nonionic surfactant in the layer increases steadily and substantially from a point below $\mathrm{T}_{3}$ to a point above the ST peak, (iv) the amount of polymer (not shown) in the layer below $\mathrm{T}_{3}$ is significantly less than for just SDS on its own, and (v) there is very little polymer at the surface above $\mathrm{T}_{4}$, unlike SDS on its own.

The lowest concentration for which there are NR results is too low for significant adsorption of free nonionic surfactant and the surface should therefore be dominated by adsorption of surface active complexes. This can be quantitatively analysed using the pseudophase approximation, for which the treatment is the same for adsorbed layers, CMCs or CACs. For a two component mixed layer the approximation gives the concentration at a fixed ST, $c_{m i x}^{\pi}$, by, ${ }^{52}$

$$
\frac{\alpha_{1}}{f_{1} c_{1}^{\pi}}+\frac{\alpha_{2}}{f_{2} c_{2}^{\pi}}=\frac{1}{c_{m i x}^{\pi}}
$$




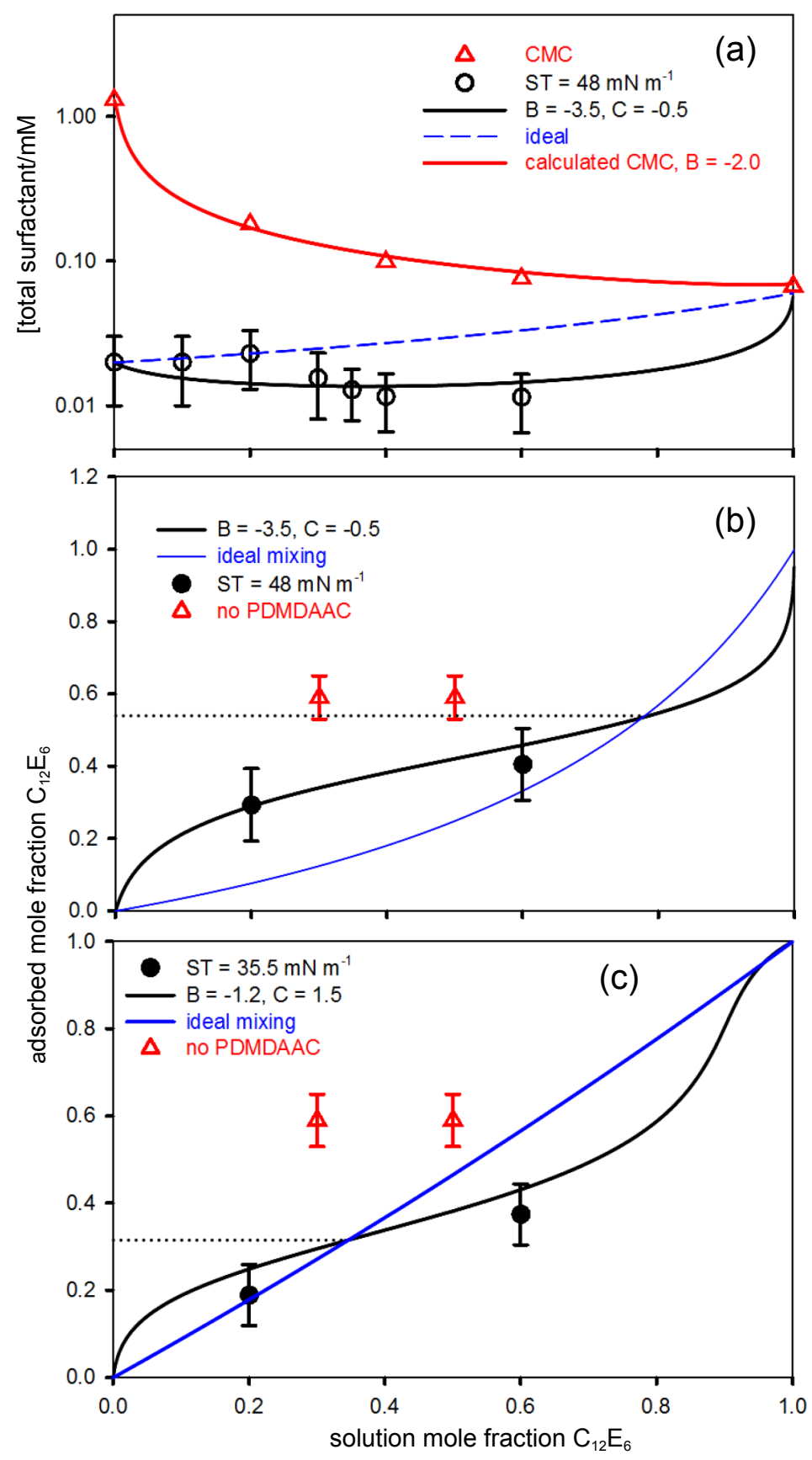

Figure 15: Fit of the pseudophase mixing model to (a) the total surfactant concentration at an $\mathrm{ST}$ of $48 \mathrm{mN} \mathrm{m}^{-1}$ of $20 \mathrm{ppm}$ PDMDAAC at different SDS: $\mathrm{C}_{12} \mathrm{E}_{6}$ compositions and the $\mathrm{CMC}$ of the corresponding surfactant mixtures, (b) the adsorbed fraction of $\mathrm{C}_{12} \mathrm{E}_{6}$ at SDS: $\mathrm{C}_{12} \mathrm{E}_{6}$ compositions of 80:20 and 40:60 at the same ST as measured by NR, and (c) the corresponding adsorbed fraction of $\mathrm{C}_{12} \mathrm{E}_{6}$ at SDS: $\mathrm{C}_{12} \mathrm{E}_{6}$ at an $\mathrm{ST}$ of $35.5 \mathrm{mN} \mathrm{m}{ }^{-1}$. The dotted lines in (b) and (c) mark the position of the minimum in the free energy of mixing on the polymer. All measurements were in $100 \mathrm{mM} \mathrm{NaCl}$, the PE-S data are from Staples et al., ${ }^{51}$ and the adsorption at the air-water interface in the absence of PDMDAAC (red triangles in (b) and (c)) are from Penfold et al. ${ }^{48}$ 
where $\alpha_{i}$ are the fractions of surfactant relative to total surfactant in the bulk solution, $f_{i}$ are the activity coefficients of the surfactants, and $c_{i}^{\pi}$ are the concentrations of the pure components at a given ST (labelled here as $\pi$, the surface pressure). The activity coefficients, $f_{i}$, in the aggregate are determined from the excess free energy of mixing and are given by ${ }^{53,54}$

$$
\ln f_{1}=B x_{2}^{2}+x_{2}^{2}\left(3-4 x_{2}\right) C+x_{2}^{2}\left(5-16 x_{2}+12 x_{2}^{2}\right) D
$$

with a parallel expression for $f_{2}$ except that the sign changes for the $C$ term. Thus the composition of the layer or adsorbed aggregate and the $c_{m i x}^{\pi}$ are entirely determined by the values of $c_{m i x}^{\pi}$ of the pure surfactants and the set of interaction parameters, $B, C$ and $D$ for the aggregate attached to the polymer. The set of values of the $c_{m i x}^{\pi}$ at a fixed ST of $48 \mathrm{mN}$ $\mathrm{m}^{-1}$ (about halfway between $\mathrm{T}_{0}$ and $\mathrm{T}_{1}$ ) were fitted by iterative solution of Eqns (11) and $(12)^{55,56}$ to give the results shown in Figure 15(a), where they are compared with the parallel calculation for mixed micellization in the absence of PDMDAAC. The polymer data require a larger attractive value of the $B$ term in the interaction than the micellization and a small negative value of the cubic term $C$ gives a better fit to the adsorption data but has little effect on the CMC data. The data is not sensitive enough to justify including the $D$ term. The effect of the cubic term $C$ is mainly to change the position of the minimum in the surface excess free energy. Thus, when $B$ and $C$ have the same sign and same value, the minimum of the excess free energy of mixing is at $1: 2 \mathrm{SDS}_{\mathrm{C}_{12}} \mathrm{E}_{6},{ }^{57}$ and when $C=0$ the minimum is at 1:1. The surface composition at the crossing point of the fitted and ideal mixing curves is the composition at this minimum. The calculated surface composition is compared with experiment at the two compositions 80:20 and 40:60, and with the corresponding adsorption in the absence of PDMDAAC, in Figure 15(b). As expected the values of the limiting $c_{i}^{\pi}$ for the single components required to fit the data show a large reduction from the corresponding $c^{\pi}$ for $\operatorname{SDS}\left(0.39 \mathrm{mM}\right.$ to $0.023 \mathrm{mM}$ at $\left.48 \mathrm{mN} \mathrm{m}^{-1}\right)$, i.e. a large binding energy of SDS to the polymer, and a small decrease from 0.07 to $0.05 \mathrm{mM}$ for $\mathrm{C}_{12} \mathrm{E}_{6}$, i.e. weak binding to the 
polymer. The minimum free energy of mixing occurs close to a 50:50 surface composition and its depth is about $25 \%$ larger than in the absence of polymer, ${ }^{48}$ but this difference is probably within the combined error of the two experiments.

The fitted results of Figure 15 are for the lowest concentration measurements in Figure 14. As the total surfactant concentration increases from this point the adsorbed mole fraction of SDS at first increases markedly up to a point between 0.05 and $0.1 \mathrm{mM}$ when it then steadily decreases to the much lower value of about 0.5 beyond the ST peak. Both effects can be attributed to a gradual exclusion of the nonionic surfactant from the complexes. First we use the pseudophase approximation to fit the $c_{m i x}^{\pi}$ and composition at a total surfactant concentration of $0.065 \mathrm{mM}$ where the $\mathrm{ST}$ is $35.5 \mathrm{mN} \mathrm{m}^{-1}$ (the third point in Figure 14). The fit to the $c_{m i x}^{\pi}$ is good but for reasons of space we only show the fit to the more sensitive composition data, which is given in Figure 15(c). Very different interaction parameters are required to fit the two sets of composition, (b) and (c) in the Figure. The minimum in the excess free energy of mixing changes from $-0.9 R T$ at the lower concentration to $-0.4 R T$ at the higher concentration but the corresponding change in the position of the minimum changes from a surface mole fraction of $0.5 \mathrm{C}_{12} \mathrm{E}_{6}$ to 0.3 . Both changes show that $\mathrm{C}_{12} \mathrm{E}_{6}$ is gradually being squeezed out of the layer and this results in some loss of surface activity of the surface complex (the mixed layer has a significantly lower ST). The loss of nonionic from the polymer is also emphasized by the much greater adsorption of the nonionic in the absence of PDMDAAC, shown as red triangles in Figure 15(b) and (c). The remaining changes can be explained by the increasing cooperativity of the bulk complex, which starts to increase the $\mathrm{ST}$, decreases adsorption, and squeezes $\mathrm{C}_{12} \mathrm{E}_{6}$ from the complexes. That the complex tends to exclude the nonionic surfactant is supported by the compositional phase diagram which shows that maximum precipitation is approximately along the charge equivalence line. ${ }^{58}$

The overall pattern of adsorption in the mixture is then that the nonionic surfactant increases the stability of the surface layer in the region where cooperative binding leads to a surface active complex. However, polymer is increasingly lost to the non-surface active 
complex, which is approximately the pure PDMDAAC-SDS complex containing no nonionic surfactant. In the 90:10 (SDS: $\mathrm{C}_{12} \mathrm{E}_{6}$ ) mixture this should cause the peak of the ST to move by $10 \%$ towards higher surfactant concentration. That it moves by significantly more than $10 \%$ (about 30\%) indicates that the extra stability that the nonionic brings to the surface complex extends the concentration range of low ST and pushes the onset of the formation of non-surface active to higher concentration. The combination of this shift and the boost to the monomer $\mathrm{C}_{12} \mathrm{E}_{6}$ concentration because of preferential fractionation of SDS into the complex means both that the ST increase is moved to higher concentration and the free surfactant ST is significantly reduced. Hence the very large suppression and the anomalous upwards shift in concentration of the ST peak clearly seen in Figure 13(c). That the increase in ST occurs significantly below the onset of precipitation emphasizes that precipitation is not directly linked to the ST rise. Finally, it is also interesting that exactly the same peculiar pattern of surface composition occurs when the same surfactant mixture interacts with branched poly(ethyleneimine)(PEI) at $\mathrm{pH} 7$ and at $\mathrm{pH} 10 .{ }^{49}$ The ST behaviour of PEI is less clear cut but again a broad but pronounced ST peak with SDS is significantly reduced when the anionic-nonionic mixture is used.

\section{Discussion}

Figure 16 compares the results of two independent sets of measurements of the ST of each of (a) NaPSS-C 12 TAB. and (b) PDMDAAC-SDS with $100 \mathrm{mM} \mathrm{NaCl}$. For both systems the ST measured by Varga and Campbell (VC) exhibits a sharp peak. VC attributed the sharp rise in ST to an equilibrium precipitation that reduces the polyelectrolyte to a low enough concentration that it can make no contribution to the ST. ${ }^{60}$ Of the two sets of data in Figure 16 the ST measurements and the precipitation measurements of VC and Staples et al. on PDMDAAC-SDS agree over the limited range measured by VC. Thus, although the rise in ST with surfactant concentration is steep, it is not a vertical edge in either set of $10 \mathrm{ppm}$ data shown in the Figure. VC's data at 100 ppm (not shown) does have a sharper change 

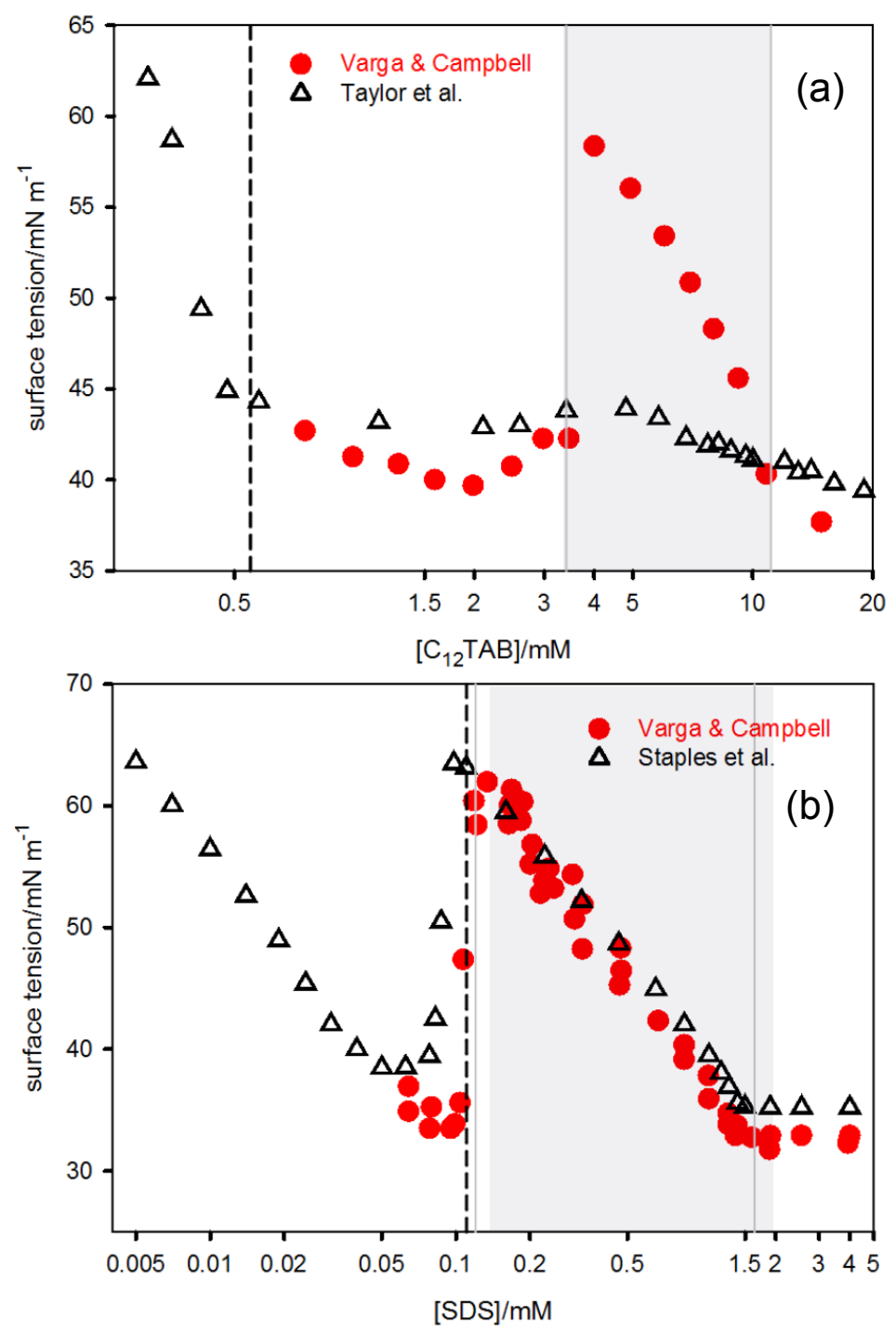

Figure 16: Surface tension measurements of (a) 100 ppm NaPSS- $\mathrm{C}_{12}$ TAB by Varga and Campbell and Taylor et al., ${ }^{12}$ and (b) 10 ppm PDMDAAC-SDS in 100 mM NaCl by Varga and Campbell ${ }^{11}$ and Staples et al. ${ }^{3}$ The shaded regions mark the long time turbidity measured by VC and the dashed lines mark the total of the free surfactant concentration and charge equivalence. The data points are redrawn from the original papers. ${ }^{25,59}$ 
in ST but this was also observed by Staples et al. The onset of precipitation, observed using turbidity, is also the same within error in the two sets of results. Apart from an anomalously low baseline ST in VC's data, the overall agreement between the two sets of data, ST and phase diagram is good. Agreement between measurements made in different ways on a system that is difficult to reproduce tends to support the conclusion that both are equilibrium results.

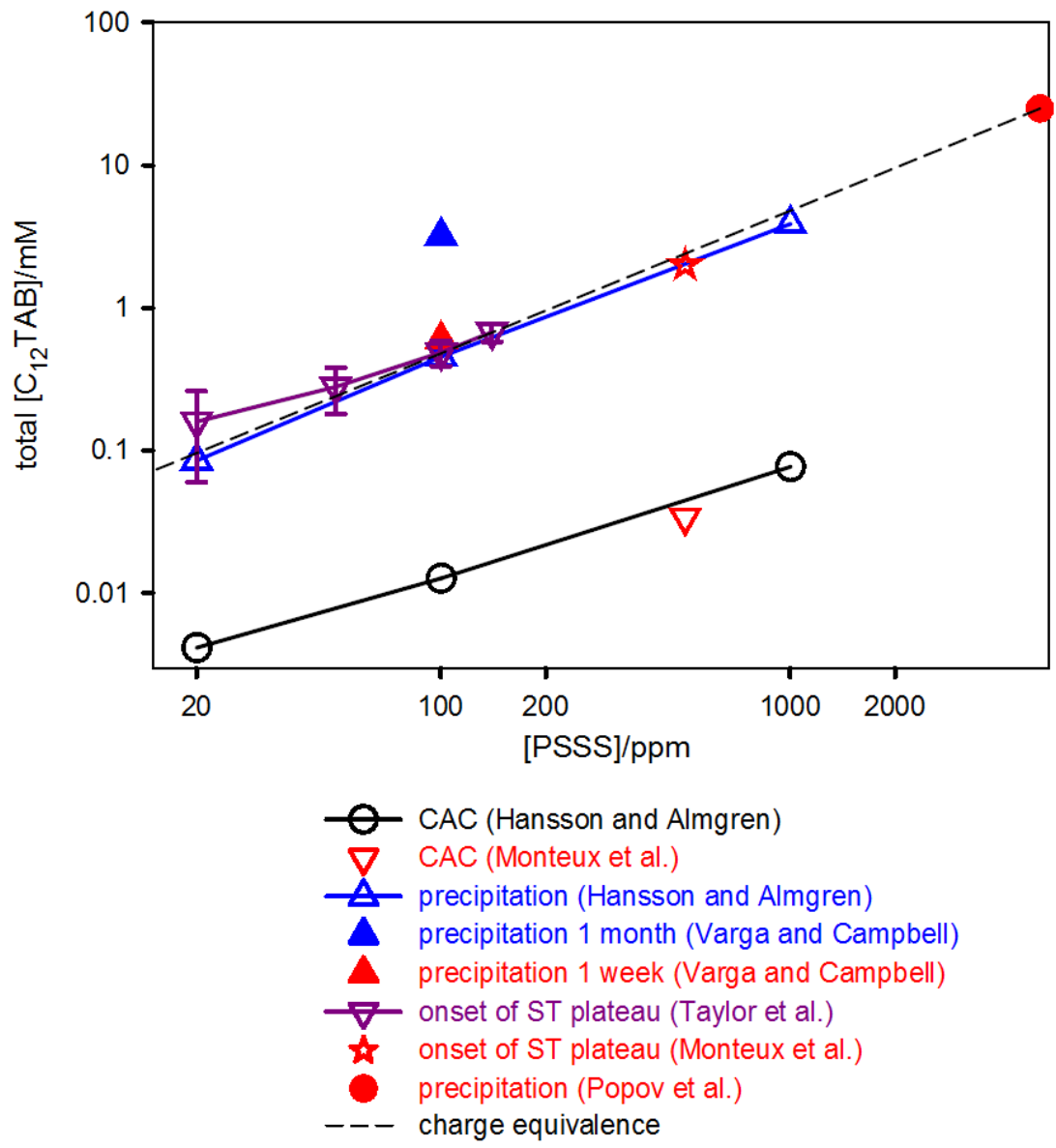

Figure 17: Values of the critical aggregation concentration (CAC) in $\mathrm{NaPSS}_{-12} \mathrm{TAB}$ mixtures, the onset of phase separation determined by Hansson and Almgren, ${ }^{24}$ the onset of precipitation and the onset of metastable precipitation at $100 \mathrm{ppm}$ determined by Varga and Campbell, ${ }^{11} \mathrm{~T}_{1}$ as measured by Taylor et al., ${ }^{12}$ a single measurement of the CAC and of $\mathrm{T}_{1}$ in $500 \mathrm{ppm} \mathrm{NaPSS},{ }^{25}$ a single measurement of precipitation at a high polymer concentration by Popov et al., ${ }^{61}$ and the theoretical charge equivalence line.

In contrast to the PDMDAAC results, there are several disagreements between VC's 
NaPSS- $\mathrm{C}_{12}$ TAB data and those measured by others. First, none of the the three other ST measurements has an ST peak. ${ }^{25,59}$ Secondly, spreading pressure measurements by Campbell et al. ${ }^{45}$ on a sample in the almost fully precipitated region give a value that is not consistent with the value of the ST at its peak (Figure 11). Thirdly, the onset of precipitation determined by $\mathrm{VC}$ is nearly an order of magnitude larger than the onset of precipitation found by Hansson and Almgren (HA), as shown in Figure 17. This discrepancy in the onset of the phase separation was not discussed in any detail by $\mathrm{VC}$, but they made a brief comment, in which they seem to attribute the discrepancy to an approximation that the concentration of bound surfactant used by HA neglects the free surfactant concentration. However, we find no evidence that such an approximation was made by HA, nor that it would have been necessary. HA's methodology is discussed further below. The values of HA's onset of precipitation occur approximately at the charge equivalence point. A fourth disagreement between $\mathrm{VC}$ and others is then that Popov et al. independently also observed precipitation at the charge equivalence point, but at a higher polymer concentration, also shown in Figure 17. A fifth disagreement is that the sharp drop in ST to a horizontal plateau at $\mathrm{T}_{1}$ (Figure 5) suggests the onset of precipitation at $\mathrm{T}_{1}$ and Monteux et al. confirmed this by identifying gel and aggregate structures in the system over a range from $T_{1}$ up to an intermediate concentration on the plateau (their Zone 2). ${ }^{25} \mathrm{~A}$ set of values of $\mathrm{T}_{1}$ was obtained by Taylor et al. and together with the value at the higher polymer concentration of Monteux et al. gives a further set of points in Figure 17 that support HA's phase diagram. VC determined their phase diagram by measurement of the turbidity. The turbidity in the early stages of precipitation gave an onset of phase separation that agrees perfectly with that of HA and this is shown as a filled red triangle in Figure 17. VC, however, attributed it to the presence of metastable particles in the system on the grounds that the turbidity between the filled red and blue triangles in Figure 17 disappears at long times. That VC's NaPSS- $\mathrm{C}_{12}$ TAB disagrees with all the several other different types of measurements suggests that their NaPSS- $\mathrm{C}_{12}$ TAB system was not fully at equilibrium, and we now examine that question in more detail. 
HA's samples for the precipitation study were prepared by putting defined amounts of each of the three components in tubes that were sealed and slowly rotated end over end for at least 2 days, centrifuged at $3500 \mathrm{rpm}$ for $10 \mathrm{~min}$, and then kept at rest for a month before spectroscopic analysis of the dilute phase for both $\mathrm{C}_{12} \mathrm{TAB}$ and NaPSS. Since they also determined the amount and the density of the dilute phase, they would have been able to determine the concentrations of both solute species in both phases. VC prepared their samples by simple initial mixing, swirling gently for a few seconds, and then transferring aliquots to small cells $\left(4 \mathrm{~cm}^{3}\right)$, where they were also left for a month before examination of their turbidity to determine the phase diagram. The difficulty with turbidity is that low levels of precipitation can be lost to the walls over time leading to a narrowing of the apparent range of precipitation. This is especially the case for small cells where the areavolume ratio is high. The analytical method used by HA would not be sensitive to this problem. The preparation of colloids is favoured by relatively quiescent conditions and VC's method of equilibration was deliberately chosen to cause as little disturbance to the sample as possible. This is unusual and more characteristic of the preparation of colloids. Most researchers routinely use more vigorous mixing methods to ensure equilibration, especially when polymers are involved, e.g. vigorous stirring, shaking or periodic rotation over an extended period, as used by HA. HA's system is therefore (a) more likely to have reached equilibrium and (b) less vulnerable to artefacts of the measurement. As shown by Campbell et al., ST and NR measurements are vulnerable to the presence of colloidal particles resulting from heterogeneous mixing. However, the possible depletion by adsorption on the very colloid prepared in the mixture (self-depletion) has not yet been considered in these systems. The experimental protocol of Varga and Campbell would seem to be optimized for self-depletion. Although Taylor et al. were clear in their original papers that there was uncertainty as to whether some of the features they observed were or were not at equilibrium, their samples were treated more vigorously than those of VC. Thus, initial stock solutions were made by mixing surfactant and polymer under vigorous stirring and left for typically a day or more. 
The final solutions used for the experiments were made by mixing such stock solutions of mixed surfactant and polymer with stock solutions of polymer, i.e. the polymer was at the same concentration in each solution. Such a final solution would typically be over a day old by the time of the NR measurement and the measuring time per sample, at that time (2001) was typically 1-2 hours and samples were in a multi-trough system awaiting measurement for several hours.

VC have argued that the ST behaviour of the PDMDAAC-SDS and NaPSS- $\mathrm{C}_{12}$ TAB systems has the same underlying explanation, which is that the onset of the ST peak coincides with the complete removal of polymer at the onset of precipitation of the PE-S complex. This leads to a step change in the ST. We have shown in the section on precipitation that a large step change in the ST is not allowed thermodynamically to accompany any phase transition. A second point is that the observed change in ST in the PDMDAAC-SDS system is not strictly vertical, i.e. it is not associated with a phase change. The third crucial observation, not mentioned by $\mathrm{VC}$, is that $\mathrm{NR}$ shows that polymer is present at the surface both sides of the peak for the PDMDAAC-SDS system, although it undergoes a dip at the peak and decreases in its coverage above the peak (Figure 12).

As shown earlier, the steep change in ST for PDMDAAC-SDS is well explained in terms of the way SDS binds to the PDMDAAC, which depends on the site binding of surfactant being much weaker than in the NaPSS- $\mathrm{C}_{12} \mathrm{TAB}$ system. This leads to strong cooperativity in the binding of the surfactant, as identified by the polymer concentration independence of both binding isotherm and initial ST behaviour. The strong cooperativity results in the initial formation of a small concentration of complexes high in surfactant. This concentration is sufficient to drive a strong decrease in ST with some rearrangement of the structure at the surface. The polymer concentration dependence of the points $T_{3}$ and $T_{4}$ in the ST shows that as further surfactant is bound the cooperativity increasingly stabilizes $3 \mathrm{D}$ bulk structures, i.e. they lose their surface activity. $\mathrm{T}_{3}$ represents the point at which the threshold concentration for monolayer adsorption of the residual surface active complexes is crossed 
and the ST therefore starts to rise rapidly. The concentration range over which this loss of surface activity occurs will be about the same as that required for the initial drop in ST approaching $\mathrm{T}_{1}$. As already discussed, the width of this transition on a log scale will appear increasingly sharp at higher concentrations. The complete loss of surface activity is reached as the polymer is saturated with surfactant and this will also be close to the condition for precipitation.

In contrast, binding in the initial NaPSS- $\mathrm{C}_{12} \mathrm{TAB}$ complex is non-cooperative, as identified by the dependence of the binding curve on polymer concentration. Non-cooperativity generally favours surface activity but the average bound fraction in this step is less than about $10 \%$, which is too low for complexes to form a low ST layer. Immediately above this initial step there is a short range of cooperative binding in NaPSS- $\mathrm{C}_{12}$ TAB, which leads to a noticeable cooperativity in the binding isotherm, but again appears either to be too low or to be too three dimensional to lower the ST. At higher bound fractions the binding isotherm changes to being non-cooperative. However, the resulting complexes do not contain sufficient surfactant for the non-cooperativity to favour a low ST until the bound fraction is relatively high, quite close to saturation at $\mathrm{T}_{1}$. A consequence of non-cooperative binding is that at any given point the complexes have a wide range of stoichiometry and the most strongly ST lowering complexes will be those close to saturation and close to precipitation. The wide range of stoichiometry also causes precipitation to be a gradual process. There is then no reason for the ST to increase at the onset of precipitation, although as the amount of added surfactant increases so does the extent of precipitation. However, thermodynamics does not allow maximum precipitation to be complete precipitation. As shown earlier, loss of surface activity can only occur if the individual complex units that make up the precipitate do not significantly lower the ST. The spreading data given in Figure 11 indicate that this does not happen in the NaPSS- $\mathrm{C}_{12}$ TAB system. Any explanation of a peak occurring somewhere well into the precipitation range can then only be a non-equilibrium one, such as the self-adsorption suggested in the section on Equilibration. The solubility of NaPSS- $\mathrm{C}_{n} \mathrm{TAB}$ 
complexes is known to decrease as the surfactant chain length $n$ increases ${ }^{62}$ and the probability of a non-equilibrium peak occurring therefore also increases with $n$. Using the same protocols for ST as for NaPSS- $\mathrm{C}_{12}$ TAB Taylor et al. observed such peaks for NaPSS with $\mathrm{C}_{14} \mathrm{TAB}$ and $\mathrm{C}_{16} \mathrm{TAB}^{63}$ Since the spreading measurements (Figure 11) indicate that the saturated complexes in both these systems spread strongly, it must be concluded that those particular ST results must also be partly non-equilibrium results, with the low concentration of the onset of the peaks occurring at the points of maximum precipitation.

\section{Conclusions}

The combination of the extensive measurements of binding isotherms, particularly their dependence on polymer concentration, with surface measurements, and the comparison of the surface and phase behaviour of $\mathrm{PE}-\mathrm{S}$ with the simpler $\mathrm{M}^{n}-\mathrm{S}$ systems, have led to a much fuller description of the equilibrium surface behaviour of $\mathrm{PE}-\mathrm{S}$ systems. A classification of the surface behaviour of PE-S complexes by ourselves about 15 years ago was based on the NR observation of multilayering and how that seemed to be associated with systems that showed no ST peak. NaPSS- $\mathrm{C}_{12}$ TAB was taken to be representative of the multilayering category and PDMDAAC-SDS-100 mM NaCl of the category with an ST peak. We have now confirmed that these two systems are quite distinct in their surface behaviour. The surfactant-polymer interaction is an order of magnitude weaker in PDMDAAC-SDS-100 $\mathrm{mM} \mathrm{NaCl}$ than in NaPSS- $\mathrm{C}_{12}$ TAB and this can be attributed, at least partly, to the lower linear charge density in PDMDAAC. ${ }^{29}$ This allows the cooperative interactions between surfactant chains to dominate the binding and surface activity. The ST of the PDMDAACSDS-100 mM NaCl is therefore at its lowest when the bound fraction is low, but it increases more or less sharply to a high value as saturation approaches. The stronger surfactantpolymer interaction in NaPSS- $\mathrm{C}_{12}$ TAB results in the dominance of non-coooperative interactions, which leads to high ST complexes at low bound fractions and low ST at saturation, close to or at precipitation. This is not accompanied by an equilibrium increase in the ST. 
Any such increase probably arises from adsorption on a high area precipitate and does not represent the true equilibrium.

The results here give some physical insight into two earlier quantitative models of the ST behaviour of PE-S mixtures. The first of these divided the complexes into a single surface active and a single non-surface active complex, in which the behaviour depended on the relative stability of the two complexes and the adsorption was calculated on the basis of mass action. ${ }^{8}$ This introduced the idea of the non-surface activity being significant. However, the second model, ${ }^{10}$ based on Butler's thermodynamic model of the surface, ${ }^{64}$ is more closely linked to the present analysis. The region of the ST curve that is most challenging to explain is between $\mathrm{T}_{1}$ and $\mathrm{T}_{4}$. Bahramian et al. emphasized the importance of the varying stoichiometry of the complexes in this region but used a relatively simple empirical device to handle the ST. The ST was taken to be approximately constant from $\mathrm{T}_{1}$ up to a lower limit where the $\mathrm{ST}$ just started to rise, i.e. effectively $\mathrm{T}_{3}$ as defined here Figure 1. The activity of the surfactant was then assumed to be a linear combination of a nondissociable (non-surface active) complex and that of the free surfactant in the case of the plateau region continuing above $\mathrm{T}_{3}$. Although this involves some further assumptions about the concentration of free surfactant at $\mathrm{T}_{3}$, the model accounts well for the variation of both the ST and the relative adsorption for the PDMDAAC-SDS system in $100 \mathrm{mM} \mathrm{NaCl}$ (Figure 4 of Bahramian et al. ${ }^{10}$ ). Part of this success is that the assumption of the linear variation of the ST between the two limits is a good approximation to almost any variation over a short range. However, the model cannot accomodate an abrupt change in the ST between $\mathrm{T}_{3}$ and $\mathrm{T}_{4}$ and therefore fails to give an adequate fit to the peaks in NaPSS-C ${ }_{14} \mathrm{TAB}$ and NaPSS $-\mathrm{C}_{16}$ TAB. We now know from the analysis here that abrupt peaks are not equilibrium peaks.

The propensity to form multilayers will be discussed in a separate paper. However, it is clear that the formation of multilayers would be structurally more favourable with (i) layer forming structures, such as are generated by non-cooperative interactions, and 
(ii) entropically more favourable adsorbing units, such as complexes with a high surfactant content. Thus, on the basis of its binding curves and its ST behaviour, NaPSS- $\mathrm{C}_{12}$ TAB is more likely to form multilayers than PDMDAAC-SDS-100 mM NaCl. The original empirical distinction between these two systems is therefore soundly based on their underlying binding characteristics, although many PE-S systems are now known not to conform closely with these two limiting cases.

\section{TOC}

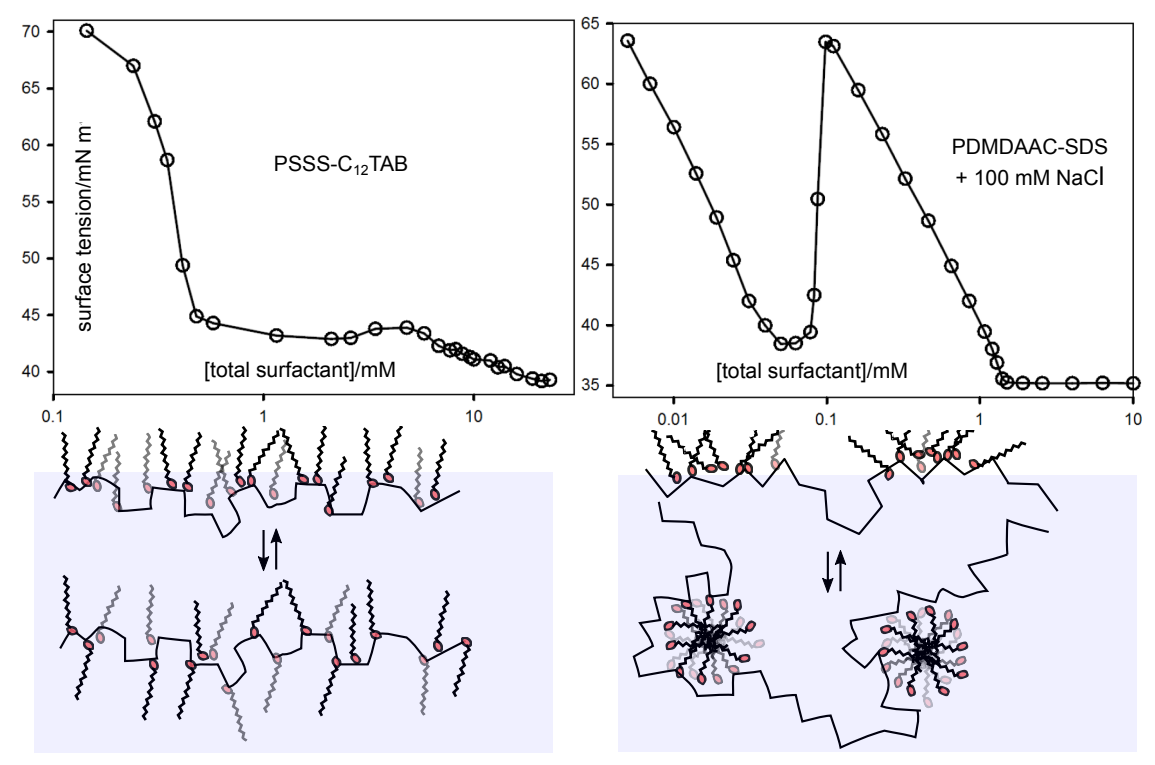

Figure 18: Surface tensions and schematic solution and surface structures of NaPSS- $\mathrm{C}_{12} \mathrm{TAB}$ and PDMDAAC-SDS-100 mM NaCl.

\section{References}

(1) Xu, H.; Penfold, J.; Thomas, R. K.; Petkov, J. T.; Tucker, I.; Webster, J. P. R. The Formation of Surface Multilayers at the Air-Water Interface from Sodium Polyethylene Glycol Monoalkyl Ether Sulfate- $\mathrm{AlCl}_{3}$ Solutions: The Role of the Size of the Polyethylene Oxide Group. Langmuir 2013, 29, 11656-11666. 
(2) Penfold, J.; Thomas, R. K.; Zhang, X. L.; Taylor, D. J. F. Nature of Amine--Surfactant Interactions at the Air-Solution Interface. Langmuir 2009, 25, 3972-3980.

(3) Staples, E.; Tucker, I.; Penfold, J.; Warren, N.; Thomas, R. K.; Taylor, D. J. F. Organization of polymer-surfactant mixtures at the air-water interface: sodium dodecyl sulfate and poly(dimethyldiallylammonium chloride). Langmuir 2002, 18, 5147-5153.

(4) Peacock, J. M.; Matijevic, E. Precipitation of Alkylbenzene Sulfonates with Metal Ions. J. Colloid Interface Sci. 1980, 77, 548-554.

(5) Meszaros, R.; Thompson, L.; Bos, M.; Varga, I.; Gilanyi, T. Interaction of sodium dodecyl sulfate with polyethyleneimine: surfactant-induced polymer solution colloid dispersion transition. Langmuir 2003, 19, 609-615.

(6) Buckingham, J. H.; Lucassen, J.; Hollway, F. Surface properties of mixed solutions of poly-L-lysine and sodium dodecyl sulfate I: equilibrium surface properties. J. Colloid Interface Sci. 1978, 67, 423-431.

(7) Su, T. J.; Styrkas, D. A.; Thomas, R. K.; Baines, F. L.; Billingham, N. C.; Armes, S. P. Neutron and X-ray Reflectivity Studies of Water-Soluble Block and Statistical Copolymers Adsorbed at the Air-Water Interface. Macromolecules 1996, 29, 6892-6900.

(8) Bell, C. G.; Breward, C. J. W.; Howell, P. D.; Penfold, J.; Thomas, R. K. Macroscopic modeling of the surface tension of polymer-surfactant systems. Langmuir 2007, 23, $6042-6052$.

(9) Bell, C. G.; Breward, C. J. W.; Howell, P. D.; Penfold, J.; Thomas, R. K. A theoretical analysis of the surface tension profiles of strongly interacting polymer-surfactant systems. J. Colloid Interface Sci. 2010, 350, 48-493.

(10) Bahramian, A.; Thomas, R. K.; Penfold, J. The Adsorption Behavior of Ionic Surfac- 
tants and Their Mixtures with Nonionic Polymers and with Polyelectrolytes of Opposite Charge at the Air-Water Interface. J. Phys. Chem. B 2014, 118, 2769-2783.

(11) Varga, I.; Campbell, R. A. General Physical Description of the Behavior of Oppositely Charged Polyelectrolyte-Surfactant Mixtures at the Air-Water Interface. Langmuir 2017, 33, 5915-5924.

(12) Taylor, D. J. F.; Thomas, R. K.; Penfold, J. The adsorption of oppositely charged polyelectrolyte-surfactant mixtures: Neutron reflection from dodecyl trimethylammonium bromide and sodium poly(styrene sulfonate) at the air-water interface. Langmuir 2002, 18, 4748-4757.

(13) Thomas, R. K.; Penfold, J. Multilayering of Surfactant Systems at the Air-Dilute Aqueous Solution Interface. Langmuir 2015, 31, 7440-7456.

(14) Xu, H.; Penfold, J.; Thomas, R. K.; Petkov, J. T.; Tucker, I.; Webster, J. P. R. The Formation of Surface Multilayers at the Air-Water Interface from Sodium Diethylene Glycol Monoalkyl Ether Sulfate- $\mathrm{AlCl}_{3}$ Solutions: The Role of the Alkyl Chain Length. Langmuir 2013, 29, 12744-12753.

(15) Xu, H.; Penfold, J.; Thomas, R. K.; Petkov, J. T.; Tucker, I.; Grillo, I.; Terry, A. Impact of $\mathrm{AlCl}_{3}$ on the Self-Assembly of the Anionic Surfactant Sodium Polyethylene Glycol Monoalkyl Ether Sulfate in Aqueous Solution. Langmuir 2013, 29, 13359-13366.

(16) Xu, H.; Penfold, J.; Thomas, R. K.; Petkov, J. T.; Tucker, I.; Webster, J. P. R.; Grillo, I.; Terry, A. Ion Specific Effects in Trivalent Counterion Induced Surface and Solution SelfAssembly of the Anionic Surfactant Sodium Polyethylene Glycol Monododecyl Ether Sulfate. Langmuir 2014, 30, 4694-4702.

(17) Li, P. X.; Li, Z. X.; Shen, H. H.; Thomas, R. K.; Penfold, J.; Lu, J. R. Application of the Gibbs equation to the adsorption of nonionic surfactants and polymers at the air- 
water interface: comparison with surface excesses determined directly using neutron reflectivity. Langmuir 2013, 29, 9335-9351.

(18) Xu, H.; Li, P. X.; Ma, K.; Thomas, R. K.; Penfold, J.; Lu, J. R. Limitations in the application of the Gibbs equation to anionic surfactants at the air-water surface: sodium dodecylsulfate and sodium dodecylmonooxyethylenesulfate above and below the CMC. Langmuir 2013, 29, 9324-9334.

(19) Li, P. X.; Thomas, R. K.; Penfold, J. Limitations in the Use of Surface Tension and the Gibbs Equation To Determine Surface Excesses of Cationic Surfactants. Langmuir 2014, 30, 6739-6747.

(20) Satake, I.; Yang, J. T. Interaction of Sodium Decyl Sulfate with Poly(L-ornithine) and Poly(L-lysine) in Aqueous Solution. Biopolymers 1976, 15, 2263-2275.

(21) Zimm, B. H.; Bragg, J. K. Theory of the Phase Transition between Helix and Random Coil in Polypeptide Chains. J. Chem. Phys. 1959, 31, 526-535.

(22) Hayakawa, K.; Kwak, J. C. I. Surfactant-polyelectrolyte interactions. 1. Binding of dodecyltrimethylammonium ions by sodium dextran sulfate and sodium poly(styrenesulfonate) in aqueous solution in the presence of sodium chloride. J. Phys. Chem. 1982, 86, 3866-3870.

(23) Almgren, M.; Hansson, P.; Mukhtar, E.; van Stam, J. Aggregation of alkyltrimethylammonium surfactants in aqueous poly(styrenesu1fonate) solutions. Langmuir 1992, 8, 2405-2412.

(24) Hansson, P.; Almgren, M. Interaction of alkyltrimethylammonium surfactants with polyacrylate and poly(styrenesulfonate) in aqueous solution: phase behavior and surfactant aggregation numbers. Langmuir 1994, 10, 2115-2124. 
(25) Monteux, C.; Williams, C. E.; Meunier, J.; Anthony, O.; Bergeron, V. Adsorption of oppositely charged polyelectrolyte-surfactant complexes at the air-water interface: formation of interfacial gels. Langmuir 2004, 20, 57-63.

(26) Nizri, G.; Lagerge, S.; Kamyshny, A.; Major, D. T.; Magdassi, S. Binding mechanism of sodium dodecyl sulfate to poly(diallyldimethylammonium chloride). J. Colloid Interface Sci. 2008, 320, 74-81.

(27) Lee, J. N.; Moroi, Y. Binding of Sodium Dodecyl Sulfate to a Cationic Polymer of High Charge Density. Bull. Chem. Soc. Japan 2003, 76, 2099-2102.

(28) Lee, J.; Moroi, Y. Investigation of the interaction between sodium dodecyl sulfate and cationic polymers. Langmuir 2004, 20, 4376-4379.

(29) Li, D. C.; Wagner, N. J. Universal Binding Behavior for Ionic Alkyl Surfactants with Oppositely Charged Polyelectrolytes. J. Am. Chem. Soc. 2014, 135, 17547-17555.

(30) Thomson, J. J. Applications of Dynamics to Physics and Chemistry; MacMillan, 1886.

(31) Chou, S. I.; Bae, J. H. Surfactant Precipitation and Redissolution in Brine. J. Colloid Interface Sci. 1983, 96, 192-203.

(32) Bozic, J.; Krznaric, I.; Kallay, N. Precipitation and micellization of silver, copper and lanthanum dodecyl sulphates in aqueous media. Colloid Polymer Sci. 1979, 257, 201205.

(33) Kallay, N.; Pastuovic, M.; Matijevic, E. Solubility and Enthalpy of Precipitation of Magnesium, Calcium, Strontium, and Barium Dodecyl Sulfates. J. Colloid Interface Sci. 1985, 106, 452-458.

(34) Alargova, R.; Petkov, J.; Petsev, D.; Ivanov, I. B.; Broze, G.; Mehreteab, A. Light Scattering Study of Sodium Dodecyl Polyoxyethylene-2-sulfate Micelles in the Presence of Multivalent Counterions. Langmuir 1995, 11, 1530-1536. 
(35) Alargova, R. G.; Danov, K. D.; Petkov, J. T.; Kralchevsky, P. A.; Broze, G.; Mehreteab, A. Sphere-to-Rod Transition in the Shape of Anionic Surfactant Micelles Determined by Surface Tension Measurements. Langmuir 1997, 13, 5544-5551.

(36) Prigogine, I.; Defay, R.; Bellemans, A.; Everett, D. H. Surface Tension and Adsorption, Chapter 6, Equation 6.7; Longman: London, 1966.

(37) Prigogine, I.; Defay, R. Chemical Thermodynamics; Longman: London, 1964.

(38) Deo, A. V.; Kulkarni, S. B.; Gharpurey, M. K.; Biswas, A. B. Rate of Spreading and Equilibrium Spreading Pressure of the Monolayers of n-Fatty Alhohols and n-Alkoxy Ethanols. J. Phys. Chem. 1962, 66, 1361-1362.

(39) IUPAC-NIST Solubility Data Series 82 Alcohol with Water-Revised and Updated. Part 5. C8 - C17 Alcohols with water. Journal of Physical Chemistry Reference Data 2007, 36,685 .

(40) Piculell, L. Understanding and exploiting the phase behavior of mixtures of oppositely charged polymers and surfactants in water. Langmuir 2013, 29, 10313-10329.

(41) Park, S. Y.; Chang, C. H.; Ahn, D. J.; Franses, E. I. Dynamic Surface Tension Behavior of Hexadecanol Spread and Adsorbed Monolayers. 1993, 9, 3640-3648.

(42) Harkins, W. D. A General Thermodynamic Theory of the Spreading of Liquids to Form Duplex Films and of Liquids or Solids to Form Monolayers. J. Chem. Phys. 1941, 9, $552-568$.

(43) Gaines, G. L. Insoluble Monolayers at Liquid-Gas interfaces; Interscience Publishers, 1966.

(44) Lee, Y. L.; Dudek, A.; Ke, T. N.; Hsiao, F. W.; Chang, C. H. Mixed PolyelectrolyteSurfactant Langmuir Monolayers at the Air-Water Interface. Macromolecules 2008, 41, 5845-5853. 
(45) Campbell, R. A.; Tummino, A.; Noskov, B. A.; Varga, I. Polyelectrolyte-surfactant films spread from neutral aggregates. Soft Matter 2016,

(46) Smith, R. D.; Berg, J. C. The collapse of surfacatnt monolayers at the air-water interface. J. Colloid Interface Sci. 1980, 74, 273-286.

(47) Gispert, M. P.; Serro, A. P.; Colac, R.; Saramago, B. Bovine serum albumin adsorption onto 316L stainless steel and alumina: a comparative study using depletion, protein radiolabeling, quartz crystalmicrobalance and atomic force microscopy. Surf. Interface Anal. 2008, 40, 1529-1537.

(48) Penfold, J.; Staples, E. J.; Thompson, L.; Tucker, I. M.; Hines, J. D.; Thomas, R. K.; Lu, J. R. Solution and Adsorption Behavior of the Mixed Surfactant System Sodium Dodecyl Sulfate-Hexaethylene Glycol Monododecyl Ether. Langmuir 1995, 11, 24962503.

(49) Penfold, J.; Tucker, I. M.; Thomas, R. K.; Taylor, D. J. F.; Zhang, J.; Bell, C. Influence of the Polyelectrolyte Poly(ethyleneimine) on the Adsorption of Surfactant Mixtures of Sodium Dodecyl Sulfate and Monododecyl Hexaethylene Glycol at the Air-Solution Interface. Langmuir 2006, 22, 8840-8849.

(50) Penfold, J.; Staples, E. J.; Tucker, I. M.; Thomas, R. K. Adsorption of Anionic and Nonionic Surfactants at the Hydrophilic Silicon Surface. Langmuir 2002, 18, 57555760.

(51) Staples, E.; Tucker, I.; Penfold, J.; Warren, N.; Thomas, R. K. Organization of polymersurfactant mixtures at the air-water interface: poly(dimethyldiallylammonium chloride), sodium dodecyl sulfate, and hexaethylene glycol monododecyl ether. Langmuir 2002, 18, 5139-5146.

(52) Rubingh, D. N. In Solution Chemistry of Surfactants; L., M. K., Ed.; Plenum Press: New York, 1979; pp 337-354. 
(53) Rowlinson, J. S.; Swinton, F. L. Liquids and Liquid Mixtures, 3rd ed.; ButterworthHeinemann Ltd: London, 1982.

(54) Barker, J. A. Determination of Activity Coefficients from Total Pressure Measurements. Australian J. Chem. 1953, 6, 207-210.

(55) Liley, J.; Thomas, R. K.; Penfold, J.; Tucker, I. M.; Petkov, J. T.; Stevenson, P.; Webster, J. R. P. Surface Adsorption in Ternary Surfactant Mixtures above the Critical Micelle Concentration: Effects of Asymmetry on the Composition Dependence of the Excess Free Energy. J. Phys. Chem. B 2017, 121, 2825-2838.

(56) Liley, J.; Thomas, R. K.; Penfold, J.; Tucker, I. M.; Petkov, J. T.; Stevenson, P.; Webster, J. R. P. The Impact of Electrolyte on Adsorption at the Air-Water Interface for Ternary Surfactant Mixtures above the Critical Micelle Concentration. Langmuir 2017, 33, 4301-4312.

(57) Li, P. X.; Ma, K.; Thomas, R. K.; Penfold, J. Analysis of the Asymmetric Synergy in the Adsorption of Zwitterionic-Ionic Surfactant Mixtures at the Air-Water Interface below and above the Critical Micelle Concentration. J. Phys. Chem. 2016, 120, 3677-3691.

(58) Staples, E. J.; Tucker, I. M.; Penfold, J.; Warren, N.; Thomas, R. K. The structure and composition of surfactant-polymer mixtures of sodium dodecyl sulphate, hexaethylene glycol monododecyl ether and poly-(dimethyldiallyl ammonium chloride) adsorbed at the air-water interface. J. Phys. Condensed Matter 2000, 12, 6023-6028.

(59) Noskov, B. A.; Loglio, G.; Miller, R. Dilational viscoelasticity of polyelectolytesurfactant adsorption films at the air-water interface: dodecyltrimethylammonium bromide and sodium poly(styrenesulfonate). J. Phys. Chem. B 2004, 108, 18615-18622.

(60) Varga, I.; Campbell, R. A. General Physical Description of the Behavior of Oppositely Charged Polyelectrolyte-Surfactant Mixtures at the Air-Water Interface, Supplementary Information. Langmuir 2017, 33, 5915-5924. 
(61) Popov, A.; Zakharova, J.; Wasserman, A.; Motyakin, M.; V, K. Macromolecular and Morphological Evolution of Poly(styrene sulfonate) Complexes with Tetradecyltrimethylammonium Bromide. J. Phys. Chem. B 2012, 116, 12332-12340.

(62) Sitar, S.; Goderis, B.; Hansson, P.; Kogej, K. Phase diagram and structures in mixtures of poly(styrenesulfonate anion) and alkyltrimethylammonium cations in water: significance of specific hydrophobic interaction. J. Phys. Chem. B 2012, 116, 4634-4645.

(63) Taylor, D. J. F.; Thomas, R. K.; Li, P. X.; Penfold, J. Adsorption of oppositely charged polyelectrolyte-surfactant mixtures: neutron reflection from alkyl trimethylammonium bromides and sodium poly(styrenesulfonate) at the air-water interface:the effect of surfactant chain length. Langmuir 2003, 19, 3712-3719.

(64) Butler, J. A. V. The Thermodynamics of the Surfaces of Solutions. Proc. Roy. Soc. A 1932, 135, 348-375. 